British Journal of Nutrition (2019), 121, 270-284

doi:10.1017/S0007114518003379

(C) The Authors 2018. This is an Open Access article, distributed under the terms of the Creative Commons Attribution-NonCommercialNoDerivatives licence (http://creativecommons.org/licenses/by-nc-nd/4.0/), which permits non-commercial re-use, distribution, and reproduction in any medium, provided the original work is unaltered and is properly cited. The written permission of Cambridge University Press must be obtained for commercial re-use or in order to create a derivative work.

\title{
Not all forms of dietary phosphorus are equal: an evaluation of postprandial phosphorus concentrations in the plasma of the cat
}

\author{
Jennifer C. Coltherd*, Ruth Staunton, Alison Colyer, Gäelle Thomas, Matthew Gilham, Darren W. Logan, \\ Richard Butterwick and Phillip Watson \\ WALTHAM Centre for Pet Nutrition, Melton Mowbray, Leicestershire LE14 4RT, UK
}

(Submitted 1 May 2018 - Final revision received 4 October 2018 - Accepted 22 October 2018)

\begin{abstract}
Phosphorus is present in diets as naturally occurring $\mathrm{P}$ from raw materials or added as an inorganic salt. However, little is known about postprandial kinetics of $\mathrm{P}$ absorption in cats. Here, we describe several studies quantifying postprandial kinetics following the ingestion of diets of varying composition. Briefly, cats were fed a meal consisting of $50 \%$ of their metabolic energy requirement in a randomised crossover design. A pre-meal baseline blood sample was taken via cephalic catheter and repeated measurements taken regularly up to $6 \mathrm{~h}$ post-meal to assess the whole blood ionised $\mathrm{Ca}$, plasma $\mathrm{P}$ and parathyroid hormone concentrations. A diet containing $4.8 \mathrm{~g}$ total P/4184 kJ (1000 kcal), $3.5 \mathrm{~g}$ P from sodium dihydrogen phosphate $\left(\mathrm{NaH}_{2} \mathrm{PO}_{4}\right) / 4184 \mathrm{~kJ}(1000 \mathrm{kcal})$ and $\mathrm{Ca}: \mathrm{P} 0.6$ caused a marked increase in plasma P from baseline to a peak of $1.976(95 \% \mathrm{CI} 1.724,2.266) \mathrm{mmol} / \mathrm{l}(P<0.001)$, whereas a diet containing $3.38 \mathrm{~g}$ total P/4184 kJ (1000 kcal), no added inorganic $\mathrm{P}$ and Ca:P 1.55 resulted in a postprandial decrease in plasma $\mathrm{P}(P=0.008)$. Subsequent data indicate that added inorganic $\mathrm{P}$ salts in the diet above $0.5 \mathrm{~g} \mathrm{P} / 4184 \mathrm{~kJ}$ (1000 kcal) cause an increase in plasma $\mathrm{P}$ in cats, while diets below this do not. The data presented here demonstrate that sources of added inorganic $\mathrm{P}$ salts cause a temporary postprandial increase in plasma $\mathrm{P}$ in a dose-dependent manner, prolonged in diets with Ca:P $<1 \cdot 0$. Dietary P derived from natural food ingredients (e.g. meat or vegetable matter) does not appear to have any effect on postprandial plasma $\mathrm{P}$.
\end{abstract}

Key words: Plasma phosphorus kinetics: Cats: Added inorganic phosphorus: Parathyroid hormone

Phosphorus is an essential nutrient, involved in several metabolic processes as well as a key component in skeletal health ${ }^{(1)}$. Broadly, dietary $\mathrm{P}$ is either present as naturally occurring $\mathrm{P}$ from protein-rich components such as meat and fish, bony material and plant ingredients or derived from the added inorganic $\mathrm{P}^{(1)}$. Studies in cats indicated that the higher the P content of the diet, the higher the amount of $\mathrm{P}$ excreted in the urine and faeces ${ }^{(2-4)}$. This relationship is not straightforward, however, as digestion and absorption of dietary $\mathrm{P}$ can be altered by a range of dietary factors $^{(5)}$. Not only does the total $\mathrm{P}$ content of the diet and the $\mathrm{Ca}: \mathrm{P}$ ratio appear to influence $\mathrm{P}$ availability, but the source of dietary $\mathrm{P}$ may also play a role in this process ${ }^{(6,7)}$. Dietary $\mathrm{P}$ derived from food ingredients, for example, poultry meal or meat and bone meal, is considered to be less bioavailable than supplemented inorganic salts; for example, P-containing mono and dibasic $\mathrm{Na}$ salts ${ }^{(3,8)}$. P-based additives are widely used in commercial food manufacturing, where they serve a number of processing functions including $\mathrm{pH}$ stabilisation, emulsification, leavening, hydration and bactericidal actions. This difference in availability is most likely due to the P contained in the organic materials being bound to in vivo proteins and intra-cellular signalling molecules, whilst added inorganic $\mathrm{P}$ is usually included in diets as a soluble salt that is able to readily disassociate and be absorbed ${ }^{(9)}$. This solubility may differ between sources of inorganic $\mathrm{P}$, for example, monophosphates readily soluble in water may lead to higher excretion of $\mathrm{P}$ in the urine compared with acid-soluble monophosphates ${ }^{(10)}$.

In healthy humans, normal circulating $\mathrm{P}$ and $\mathrm{Ca}$ concentrations are maintained via modulation of calcitrol (1,25-dihydroxyvitamin $\mathrm{D}_{3}$ ), fibroblast growth factor (FGF-23) and parathyroid hormone $(\mathrm{PTH})^{(11)}$. During prolonged exposure to a high dietary $\mathrm{P}$ intake in humans, FGF-23 production is stimulated; this down-regulates the expression of renal sodium-phosphate co-transporters and decreases 1,25-dihydroxyvitamin $\mathrm{D}_{3}$ levels, leading to increased $\mathrm{P}$ excretion through the kidneys ${ }^{(12)}$. In cats, dogs and rats, it has been observed that acutely after a meal, an increase in serum $\mathrm{P}$ causes a reduction in ionised $\mathrm{Ca}$ (iCa) that in turn increases PTH secretion, also leading to decreased resorption of $\mathrm{P}$ in the kidney and increased excretion into the urine $^{(13,14)}$. A substantial reduction in kidney function can also impair the regulation of phosphate balance, resulting in chronically elevated circulating phosphate concentrations in humans ${ }^{(15)}$. This response has been associated with cardiovascular events, cardiovascular mortality and all-cause mortality as well

Abbreviations: FGF-23, fibroblast growth factor 23; iCa, ionised calcium; PTH, parathyroid hormone; SDHP, sodium dihydrogen phosphate.

* Corresponding author: Dr J. C. Coltherd, email jen.coltherd@effem.com 
as with human patients having chronic kidney disease $(\mathrm{CKD})^{(16)}$.

Dobenecker et al. ${ }^{(6)}$ recently showed that feeding a high-P diet, with a low Ca:P, to cats for $29 \mathrm{~d}$ caused glucosuria and microalbuminuria with a decreased creatinine clearance; all of which are indicators of kidney dysfunction ${ }^{(6)}$. The use of phosphate supplements as low as $0.5 \%$ by DM weight of diet has been shown to cause renal and vascular calcification in adult rats ${ }^{(14,17)}$. In addition, the feeding of semi-purified diets to rats has been shown to result in morphological changes to the kidneys, consistent with the disease, when compared with diets comprising $\mathrm{P}$ from a mostly natural source ${ }^{(18)}$. These observations are consistent with the literature suggesting that the absorption of natural sources of $\mathrm{P}$ is considerably lower than inorganic phosphate salt supplements ${ }^{(6,17,19)}$. Recent work from our group found that cats fed diets containing total P of $4 \cdot 8 \mathrm{~g} / 4184 \mathrm{~kJ}$ (1000 kcal), of which $3.5 \mathrm{~g} \mathrm{P} / 4184 \mathrm{~kJ}(1000 \mathrm{kcal})$ was in the form of inorganic sodium dihydrogen phosphate (SDHP) and $\mathrm{Ca}: \mathrm{P}=0.6$ for 4 weeks, developed irreversible kidney changes characterised by reduced glomerular filtration rate, elevated serum creatinine and urinary albumin:creatinine ratio and evidence of structural abnormalities within the kidney ${ }^{(20)}$. In a follow-up study, adult cats fed a diet with P from SDHP inclusion of $1.5 \mathrm{~g} / 4184 \mathrm{~kJ}(1000 \mathrm{kcal})$, $3.6 \mathrm{~g}$ total $\mathrm{P} / 4184 \mathrm{~kJ}(1000 \mathrm{kcal})$ and $\mathrm{Ca}: \mathrm{P}=0.9$ for over 28 weeks showed no mean changes in blood biochemistry indicative of renal dysfunction; however, there was sonographic evidence indicative of new renoliths and renal structural changes in some cats ${ }^{(20)}$. There were three exceptions in the test group, where cats developed signs of renal dysfunction towards the end of the feeding period, with clinically relevant changes in blood biochemistry apparent from week 21 of feeding. Therefore, there is a need to better understand dietary $\mathrm{P}$ absorption.

Although there are data for postprandial serum or plasma $\mathrm{P}$ in cats ${ }^{(8)}$ and $\operatorname{dogs}^{(21)}$, these measures and the associated regulatory responses have been examined in greater detail in healthy humans as well as in humans with $\mathrm{CKD}^{(22)}$. Dietary management of CKD aims to limit $\mathrm{P}$ load on the kidneys through a reduction in $\mathrm{P}$ intake, but various strategies are also applied to reduce bioavailability, including the use of phosphate binding medications, as well as dietary manipulation by applying different $\mathrm{P}$ sources ${ }^{(23,24)}$. The findings of these studies in humans demonstrate that ingestion of added inorganic $\mathrm{P}$ increased serum phosphate concentrations in one study ${ }^{(24)}$ and that plasma $\mathrm{P}$ decreased following consumption of vegetarian diets in another ${ }^{(23,24)}$, highlighting that postprandial plasma $\mathrm{P}$ kinetics are different when different sources of $\mathrm{P}$ are ingested. Karp et $a l{ }^{(25)}$ found similar trends in serum phosphate and urinary $\mathrm{P}$ excretion, when tripolyphosphate and monophosphate supplements were consumed. In addition, healthy male volunteers fed a $500 \mathrm{mg} \mathrm{P}$ supplement showed that serum phosphate increased postprandially, while a transient decrease was apparent after a placebo ${ }^{(26)}$. Sustained increases in plasma $\mathrm{P}$ are thought to be harmful due to the physiological consequences associated with the regulation of plasma $\mathrm{P}^{(14)}$. There is a suggestion that over time, increased FGF-23 can lead to a loss of $\mathrm{Ca}$-dependent inhibition of PTH, and suppression of PTH by FGF-23 itself, which in turn could lead to damage to organs such as the kidney and parathyroid gland ${ }^{(27,28)}$.
To date relatively little work has been undertaken to understand the factors affecting postprandial plasma $\mathrm{P}$ concentrations in cats. This knowledge is vital, particularly as evidence is accumulating to suggest that dietary P may contribute to the aetiology of diseases such as CKD or CVD. We ${ }^{(20)}$ and others (Dobenecker et al. $^{(6)}$; Pastoor et $a l^{(3)}$ ) have reported changes in kidney function following exposure to diets containing high levels of $\mathrm{P}$, but the mode of action, time course and clinical significance of the findings make drawing firm conclusions on the role of dietary $\mathrm{P}$ difficult to establish. Responses to differences in dietary $\mathrm{P}$ levels as well as differing bioavailability of various sources of dietary $\mathrm{P}$ present in the diets have been determined in cats and dogs ${ }^{(3,6,21,29)}$. With this in mind, we describe herein a series of studies designed to evaluate the postprandial profiles of $\mathrm{P}$ in the plasma of cats following a range of dietary interventions.

\section{Methods}

\section{Overview}

A series of randomised crossover studies were performed, as detailed in Table 1 . These studies involved a single exposure to each test diet comprising $50 \%$ of the individuals' metabolic energy requirement (MER), and subsequent monitoring of the postprandial plasma responses for $6 \mathrm{~h}$ post-ingestion. Diets were blinded for feeders and during all sample analyses. The number of cats in each study varied based on separate powering for individual study. The timings of blood sampling and blood volumes were refined as the series of studies progressed; there was a minimum of $7 \mathrm{~d}$ between sampling occasions for each study. Each research protocol was reviewed and approved by the WALTHAM Animal Welfare and Ethical Review Body and carried out under the authority of the revised Animals (Scientific Procedures) Act 1986 incorporating EU Directive 2010/63. Cats were extensively trained for and habituated to all procedures.

\section{Cats}

All cats utilised throughout the studies were neutered. To ensure eligibility for the study, all cats underwent a pre-study health assessment, including a physical examination and haematological, plasma biochemical and urine analysis. Cats were group housed in social rooms, except during $24 \mathrm{~h}$ urine collection periods when, to facilitate sample collection, they were individually housed in lodges. Throughout the study, diets were offered in amounts to maintain an ideal body condition score according to a 9-point scale $^{(30)}$ and deionised water was available ad libitum at all times on the study day; a second meal (50\% MER) was offered following the final sample collection. This second meal was a single batch of a fully complete and balanced commercial diet that was compliant with Association of American Feed Control Officials (AAFCO) and European Pet Food Industry Federation (FEDIAF) guidelines. This was analysed to calculate the total P intake over $24 \mathrm{~h}$.

\section{Diets}

For these studies, experimental dry and wet diets were specifically formulated and manufactured at Royal Canin and Mars Inc., respectively. Nutritional composition of the diets was confirmed 
Table 1. Study details

(Mean values and ranges)

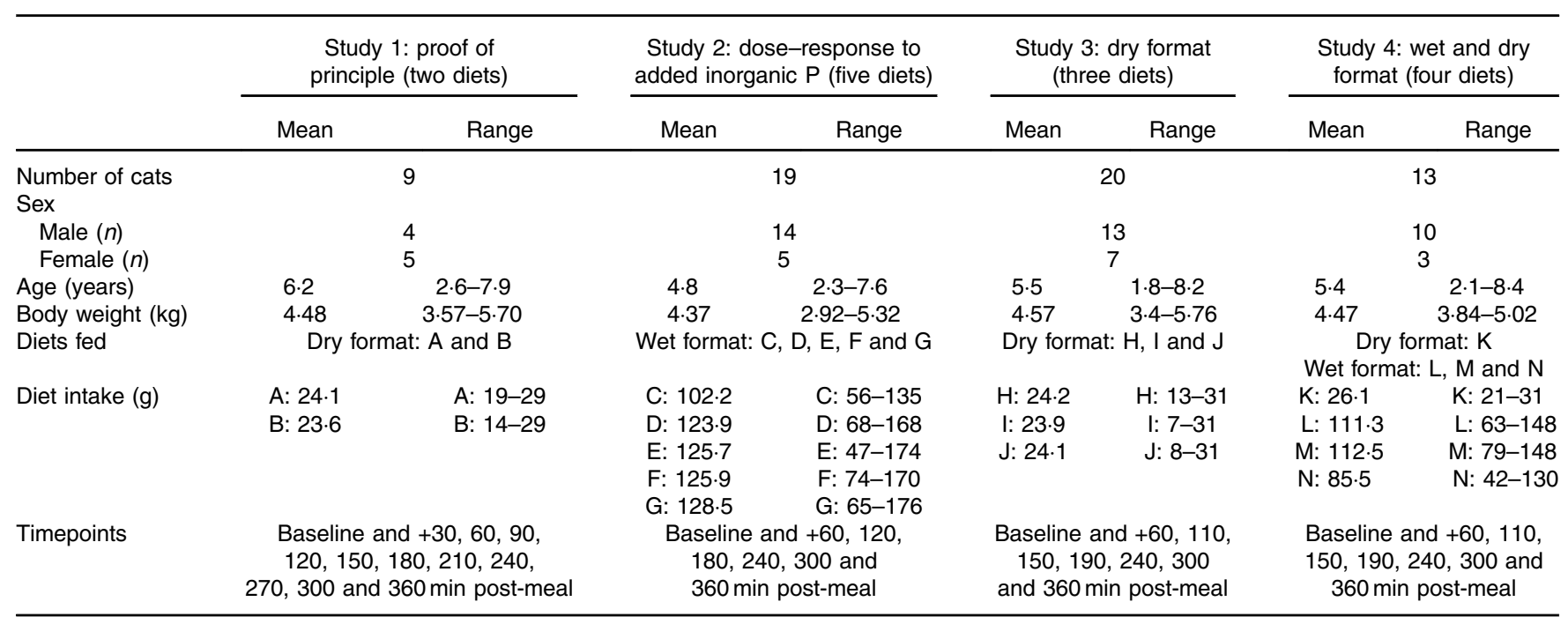

through nutritional analyses carried out at Eurofins Ltd, Wolverhampton, UK, utilising Association of Official Analytical Chemists official methods of analyses (see Table 2 for details).

\section{Blood sampling}

Following an overnight fast, cats were given topical local anaesthesia (1 $\mathrm{ml} \mathrm{EMLA}^{\mathrm{TM}}$ cream 5\%; AstraZeneca) and a 22G cephalic catheter was positioned, which remained in place for the duration of the sampling period. A $0.2 \mathrm{ml}$ heparin flush and discard was performed before sampling and a $2 \mathrm{ml}$ saline flush followed each sample to maintain patency. A fasted blood sample was collected to provide a baseline, with further samples taken at intervals described in each specific study up to, and including, $6 \mathrm{~h}$ post-feeding.

\section{Blood sample processing and analysis}

Blood samples $(0.5 \mathrm{ml})$ were collected into lithium heparin tubes and kept on ice until analysis was performed within 60 min of sampling. iCa was measured in whole blood using a Stat Profile Prime Veterinary Critical Care Analyser (Nova Biomedicals). Performance of the instrument was verified by analysis of tri-level quality control material before and after sample measurement via an ion-selective electrode. Plasma was isolated by centrifugation of the remaining blood samples at $2000 \mathrm{~g}$ for $10 \mathrm{~min}$ at $4^{\circ} \mathrm{C}$. P, in the form of orthophosphate, was photometrically quantified on non-ashed plasma using an AU480 clinical chemistry analyser (Beckman Coulter) according to the manufacturer's instructions and concentrations reported in $\mathrm{mmol} / \mathrm{l}$. Bi-level quality control material before, midway and after sample day measurements was used to confirm acceptable instrument performance.

Analysis of plasma PTH and FGF-23 were performed at the Royal Veterinary College, London. Blood samples ( $1 \mathrm{ml}$ for each measure) were collected in EDTA containing tubes and plasma obtained by centrifugation at $2000 \mathrm{~g}$ for $10 \mathrm{~min}$ at $4^{\circ} \mathrm{C}$, before being stored at $-80^{\circ} \mathrm{C}$ until analysis. Intact plasma FGF-23 was measured using a human sandwich ELISA (Kainos Laboratories), as previously described ${ }^{(31)}$, and PTH concentrations by a RIA previously validated for use with feline samples ${ }^{(32)}$.

\section{Urine sample analysis}

All urine passed over $24 \mathrm{~h}$ following the feeding of the test diet were collected to assess renal $\mathrm{P}$ excretion. Cats were individually housed during this $24 \mathrm{~h}$ collection period, and urine was collected in a litter tray containing hydrophobic sand or glass beads. Urine samples were acidified to $\mathrm{pH} 2( \pm 0 \cdot 1)$ using concentrated hydrochloric acid, and centrifuged at $2000 \mathrm{~g}$ for $5 \mathrm{~min}$ at $4^{\circ} \mathrm{C}$ before $\mathrm{P}$ analysis was undertaken on the AU480 analyser as described above.

\section{Statistical powering}

Following completion of a pilot study, sample size analyses were performed to investigate the number of cats needed in a crossover study design, with 2,3 or 4 diets, to detect fold changes with at least $80 \%$ power in plasma P levels of $5-20 \%$ when measuring 6,8 or 10 time points. Plasma $\mathrm{P}$ levels from this pilot study were used to estimate the betweencat variability $\left(\mathrm{SD}=0.0261 \log _{10} \mathrm{mmol} / \mathrm{l}\right)$, between-diet withincat variability $\left(\mathrm{SD}=0.0393 \log _{10} \mathrm{mmol} / \mathrm{l}\right)$ and within diet within cat variability $\left(\mathrm{SD}=0.0395 \log _{10} \mathrm{mmol} / \mathrm{l}\right)$. The baseline pre-feed levels were also estimated to be $0.0816 \log _{10} \mathrm{mmol} / \mathrm{l}$. Data were then simulated (1000 for each scenario) according to these variability estimates and by inducing a difference for one diet at one time point. The analysis was performed, as per the postprandial pilot data analysis (given below). The power was estimated by the percentage of simulations where all comparisons relative to the mean that was changed were significant, at the overall family-wise error rate of $5 \%$. 
Table 2. Nutrient composition of the diets

\begin{tabular}{|c|c|c|c|c|c|c|c|c|c|c|c|c|c|c|}
\hline \multirow[b]{2}{*}{ Diet } & \multicolumn{2}{|c|}{ Study 1} & \multicolumn{5}{|c|}{ Study 2} & \multicolumn{3}{|c|}{ Study 3} & \multicolumn{4}{|c|}{ Study 4} \\
\hline & A & B & C & D & $\mathrm{E}$ & $\mathrm{F}$ & G & $\mathrm{H}$ & 1 & $\mathrm{~J}$ & $\mathrm{~K}$ & L & M & $\mathrm{N}$ \\
\hline Total P (g/4184 kJ (1000 kcal)) & 4.78 & $3 \cdot 38$ & $1 \cdot 13$ & 4.02 & 4.48 & 4.48 & 4.90 & 3.79 & 3.86 & 3.52 & 3.59 & $4 \cdot 17$ & 5.50 & 4.85 \\
\hline Inorganic $\mathrm{P}^{*}(\mathrm{~g} / 4184 \mathrm{~kJ}(1000 \mathrm{kcal}))$ & $\begin{array}{c}3.50 \\
\text { (SDHP) }\end{array}$ & 0 & 0 & $\begin{array}{c}0.50 \\
\text { (STPP) }\end{array}$ & $\begin{array}{c}0.75 \\
\text { (STPP) }\end{array}$ & $\begin{array}{c}1.00 \\
\text { (STPP) }\end{array}$ & $\begin{array}{c}1.50 \\
\text { (STPP) }\end{array}$ & 0 & $\begin{array}{c}1.50 \\
\text { (SDHP) }\end{array}$ & $\begin{array}{c}1.50 \\
\text { (STPP) }\end{array}$ & $\begin{array}{c}1.50 \\
\text { (SDHP) }\end{array}$ & $\begin{array}{c}1.50 \\
\text { (STPP) }\end{array}$ & $\begin{array}{c}1.50 \\
\text { (STPP) }\end{array}$ & $\begin{array}{c}0.50 \\
\text { (STPP) }\end{array}$ \\
\hline Ca (g/4184 kJ (1000 kcal)) & $2.60 \dagger$ & 5.23 & 1.38 & 6.54 & $7 \cdot 12$ & 6.62 & 8.03 & 6.07 & $6 \cdot 14$ & 6.00 & 3.34 & 7.85 & $9 \cdot 36$ & $7 \cdot 12$ \\
\hline Ca:P ratio & 0.50 & 1.55 & 1.23 & 1.63 & 1.59 & 1.48 & 1.64 & 1.60 & 1.59 & 1.70 & 0.93 & 1.88 & 1.70 & 1.47 \\
\hline $\mathrm{Na}(\mathrm{g} / 4184 \mathrm{~kJ}(1000 \mathrm{kcal}))$ & 3.33 & 1.71 & 1.17 & 1.76 & $2 \cdot 13$ & $2 \cdot 19$ & $3 \cdot 19$ & 1.72 & 1.73 & 2.46 & 1.35 & 2.63 & 2.69 & 1.48 \\
\hline $\operatorname{Mg}(\mathrm{g} / 4184 \mathrm{~kJ}(1000 \mathrm{kcal}))$ & $0 \cdot 18$ & 0.29 & 0.09 & 0.22 & 0.22 & 0.21 & 0.22 & 0.28 & 0.24 & 0.22 & 0.28 & 0.23 & 0.24 & 0.32 \\
\hline $\mathrm{K}(\mathrm{g} / 4184 \mathrm{~kJ}(1000 \mathrm{kcal}))$ & $2 \cdot 29$ & 2.03 & 2.51 & 3.43 & 3.57 & 3.39 & 3.55 & 1.95 & 2.08 & 1.81 & $2 \cdot 27$ & $2 \cdot 76$ & 2.79 & 2.83 \\
\hline Chloride (g/4184 kJ (1000 kcal)) & 1.65 & 3.25 & 3.59 & 4.21 & 4.23 & 2.07 & $2 \cdot 01$ & $3 \cdot 19$ & 1.74 & 1.72 & 1.39 & $2 \cdot 27$ & 2.33 & 2.03 \\
\hline Vitamin $D_{3}(\mu \mathrm{g} / 4184 \mathrm{~kJ}(1000 \mathrm{kcal}))$ & 4.57 & $6 \cdot 31$ & 5.92 & 7.37 & 7.63 & 7.47 & 7.73 & $7 \cdot 20$ & 6.69 & 6.02 & 4.59 & 6.63 & 6.73 & 22.75 \\
\hline Protein $(\mathrm{g} / 4184 \mathrm{~kJ}(1000 \mathrm{kcal}))$ & $61 \cdot 13$ & 89.44 & $100 \cdot 63$ & $131 \cdot 38$ & $131 \cdot 28$ & 131.93 & $131 \cdot 33$ & 84.52 & $91 \cdot 18$ & 86.55 & 64.27 & 98.57 & $108 \cdot 46$ & $107 \cdot 88$ \\
\hline Fat $(\mathrm{g} / 4184 \mathrm{~kJ}(1000 \mathrm{kcal}))$ & 39.88 & 37.96 & 62.57 & 48.47 & $46 \cdot 23$ & 47.86 & $45 \cdot 52$ & $39 \cdot 25$ & 34.06 & $40 \cdot 15$ & $42 \cdot 61$ & 48.72 & 53.65 & 71.56 \\
\hline Ash (g/4184 kJ (1000 kcal)) & 28.07 & 24.96 & 11.28 & 28.06 & $30 \cdot 38$ & 47.86 & 38.82 & 26.95 & 27.89 & $28 \cdot 15$ & 21.53 & 28.89 & $32 \cdot 19$ & $27 \cdot 77$ \\
\hline Crude fibre (g/4184 kJ (1000 kcal)) & $5 \cdot 20$ & 7.80 & 3.08 & 2.55 & 2.64 & 2.59 & 2.68 & 8.64 & 8.58 & 7.04 & 7.49 & 3.40 & $1 \cdot 17$ & 7.48 \\
\hline Moisture $(\mathrm{g} / 100 \mathrm{~g})$ & $5 \cdot 76$ & 4.90 & 81.00 & 82.50 & $82 \cdot 70$ & $82 \cdot 36$ & $82 \cdot 24$ & $4 \cdot 70$ & $4 \cdot 70$ & $5 \cdot 20$ & $4 \cdot 31$ & 79.66 & 80.90 & $80 \cdot 80$ \\
\hline PME $\neq(\mathrm{kJ} / 100 \mathrm{~g})$ & 1594.52 & $1599 \cdot 12$ & 407.94 & 328.03 & $316 \cdot 73$ & 323.42 & 312.54 & $1599 \cdot 12$ & $1560 \cdot 21$ & 1604.98 & $1647 \cdot 07$ & 369.45 & 358.57 & 391.62 \\
\hline Food base excess§ (mEq/kg) & -0.56 & $2 \cdot 37$ & -0.16 & 0.64 & 0.77 & 1.03 & 1.30 & 2.80 & 4.38 & 6.01 & -0.44 & 1.95 & 1.83 & $1 \cdot 12$ \\
\hline
\end{tabular}

SDHP, sodium dihydrogen phosphate; STPP, sodium tripolyphosphate; PME, predicted metabolisable energy.

* Inorganic $\mathrm{P}$ represents the target $\mathrm{P}$ inclusion level from: $\mathrm{SDHP}, \mathrm{NaH}_{2} \mathrm{PO}_{4}$ or $\mathrm{STPP}, \mathrm{Na}_{5} \mathrm{P}_{3} \mathrm{O}_{10}$.

† Same diet as fed in Alexander et al. ${ }^{20}$ without the $200 \mathrm{mg} / 4184 \mathrm{~kJ}(1000 \mathrm{kcal}) \mathrm{Ca}$ suppla

$\S$ Food base excess calculated from methionine and cysteine according to Jeremias et al ${ }^{(61)}$ on a per kg food basis. 


\section{Statistical analysis}

For each study, the postprandial plasma $\mathrm{P}$ levels (primary measure), iCa, PTH and FGF-23 were analysed using linear mixed effects models (LMM), with time nested in diet nested in animal as the random effects and diet, time point and their interaction as the categorical fixed effects. Plasma P, PTH and FGF-23 data were $\log _{10}$ transformed before analyses where increasing variation in residuals with increasing value was observed. For some measures, data were missing because samples were not taken or of failure of laboratory equipment; however, the restricted maximal likelihood (REML) method of analysis was used to account for this and assumed these values were missing at random. Comparisons were made, using a family-wise adjusted $5 \%$ level for each measure, between diets at each time point and between fasted to each subsequent postprandial time point for each diet. Accordingly, means, differences between means or fold changes were reported with $95 \%$ family-wise CI. In addition, for each cat on each diet in each study, the plasma $\mathrm{P}$ at baseline was subtracted from all post-baseline time points, and the trapezium rule was then used to calculate the AUC in $\mathrm{mmol} / \mathrm{l} \times \mathrm{h}$. Separate LMM were fit for each trial with AUC as the response. Distributional assumptions were assessed by visual inspection of the residuals and were deemed to be upheld for all models. The fixed effect for each model was diet, and the random effect was diet nested in animal. From each model, mean AUC with 95\% CI are reported for all diets. Comparisons between diets are reported as differences with $95 \% \mathrm{CI}$ and $P$ values.

Urinary $\mathrm{P}$ excretion was analysed by LMM with cat as a random effect and diet as a fixed effect. A comparison was made between diets using the $5 \%$ level. Means and differences between means are reported with 95\% CI.

Analyses were performed in statistical software R version 3.3.2 $2^{(33)}$ using libraries $1 m e 4^{(34)}$ for LMM, multcomp ${ }^{(35)}$ for simultaneous inference and ggplot ${ }^{(36)}$ for data visualisation.

\section{Results}

Study 1 - proof of principle: can postprandial plasma phosphorus profiles be manipulated by different diet compositions?

Diet A significantly increased plasma P to a peak of 1.98 (95\% CI $1.72,2.27) \mathrm{mmol} / \mathrm{l}$ at $150 \mathrm{~min}$ where it plateaued before gradually decreasing during the final three time points $(P<0 \cdot 001$; Fig. 1(a)). Diet B showed a significant decrease from baseline in plasma P from 90 until $360 \mathrm{~min}(P \leq 0 \cdot 008$; Fig. $1(\mathrm{a})$ ). Diet A had a significantly higher AUC of 3.65 (95\% CI $2 \cdot 8,4.49) \mathrm{mmol} / \mathrm{l} \times \mathrm{h}$ than diet $\mathrm{B}$ which had a negative AUC of $-1 \cdot 1$ (95\% CI $-1 \cdot 4$, $-0.621) \mathrm{mmol} / \mathrm{l} \times \mathrm{h}(P<0.001$; Fig. 2 and Table 3$)$. The increase in plasma $\mathrm{P}$ in cats fed diet A was accompanied by a significant reduction in iCa compared with diet B at 300 and 360 min $(P<0.001$ and $P=0.007$, respectively; Fig. 1(b)). An increase in PTH was also observed from 120 min onwards when cats were fed diet A $(P<0 \cdot 001$; Fig. 1(c)). There was no observed change in PTH for cats fed diet B ( $P \geq 0.998$; Fig. 1(c)). No differences in FGF-23 were observed between diets or from baseline over the $6 \mathrm{~h}$ postprandial sampling period (Fig. $1(\mathrm{~d})$ ).
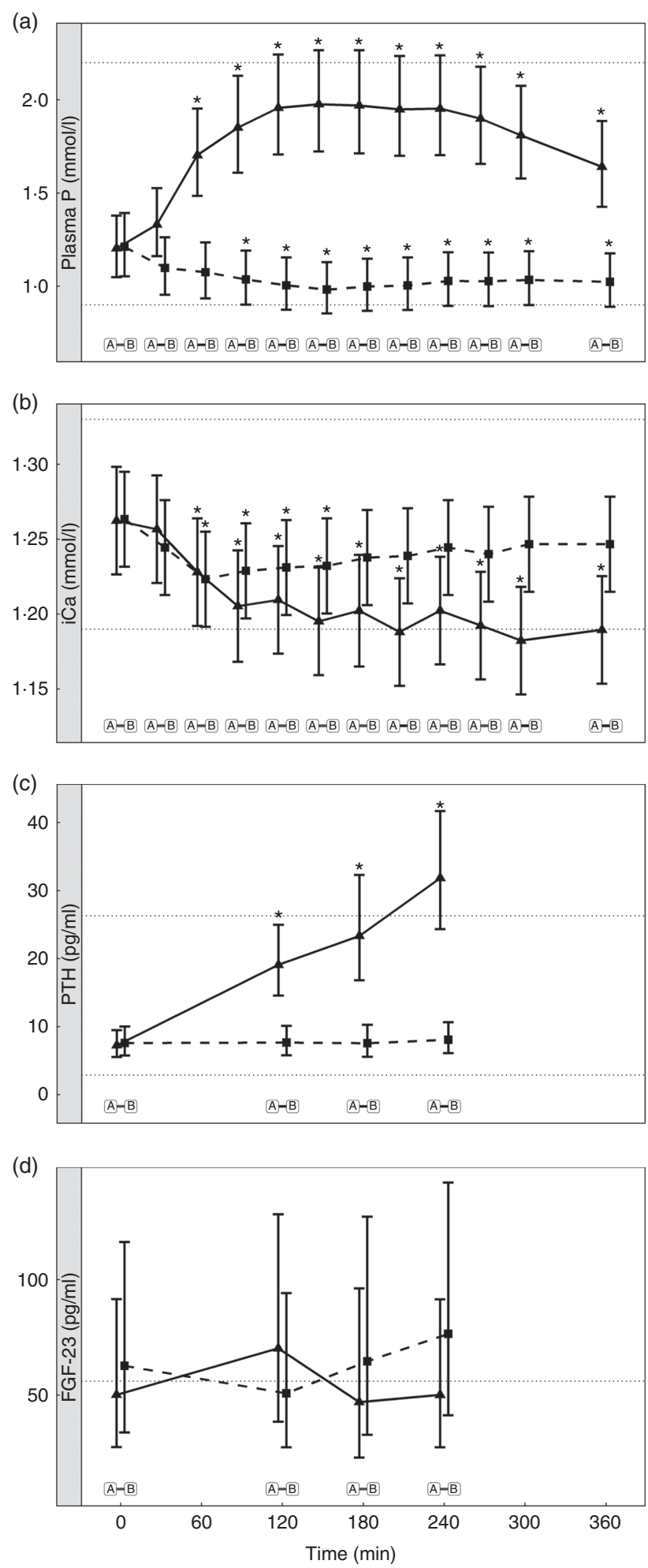

Fig. 1. Biochemical parameters over the study day for study 1. (a) Plasma phosphorus, (b) whole blood ionised calcium (iCa), (c) plasma parathyroid hormone (PTH) and (d) fibroblast growth factor 23 (FGF-23). Diet A (—-): $4.8 \mathrm{~g}$ total phosphorus $/ 4184 \mathrm{~kJ}(1000 \mathrm{kcal}), 3.5 \mathrm{~g}$ phosphorus from sodium dihydrogen phosphate/4184 kJ (1000 kcal), calcium:phosphorus 0.56. Diet B (- $\quad-): 3.38 \mathrm{~g}$ total phosphorus/4184 kJ (1000 kcal), no added inorganic phosphorus, calcium:phosphorus 1.55 . Values are means, with $95 \% \mathrm{Cl}$ represented by vertical bars. ${ }^{*}$ Mean values were significantly different from baseline $(P<0.05)$. Reference ranges are shown for each parameter, but for FGF-23 only the minimum is shown. Significant differences between diets: false $(\square)$ and true $(\square)$. 


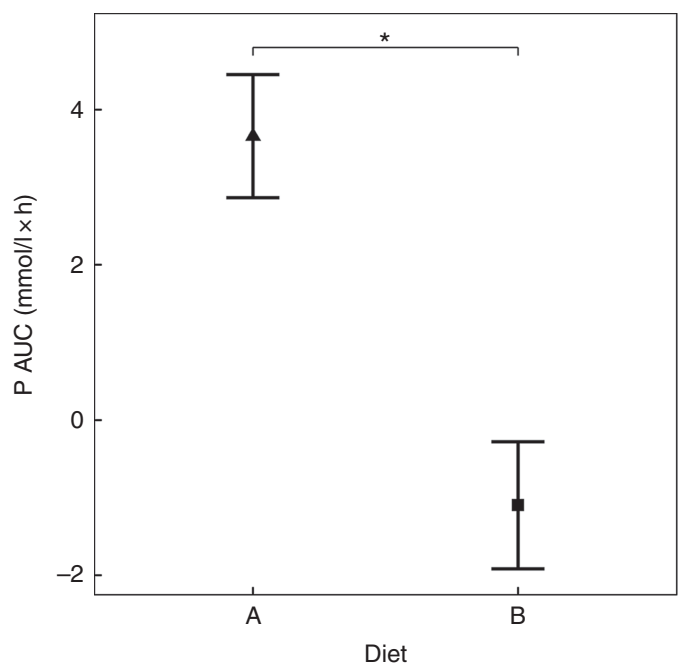

Fig. 2. AUC data for study 1. Diet $A(\mathbf{\Lambda}): 4.8 \mathrm{~g}$ total phosphorus $/ 4184 \mathrm{~kJ}(1000$ $\mathrm{kcal}), 3.5 \mathrm{~g}$ phosphorus from sodium dihydrogen phosphate/4184 kJ (1000 kcal), calcium:phosphorus 0.56 . Diet B (口): $3.38 \mathrm{~g}$ total phosphorus $/ 4184 \mathrm{~kJ}$ (1000 kcal), no added inorganic phosphorus, calcium:phosphorus 1.55. Values are means, with $95 \% \mathrm{Cl}$ represented by vertical bars. * Mean values were significantly different between diets $(P<0.05)$.

Urinary $\mathrm{P}$ excretion increased significantly when cats were fed diet A (Table 3) $(P<0 \cdot 001)$.

\section{Study 2 - does added inorganic phosphorus in the diet} drive the postprandial increase in plasma phosphorus in a dose-dependent manner?

Inorganic $\mathrm{P}$ inclusion increased peak plasma $\mathrm{P}$ concentrations in a dose-dependent manner. A significant increase from baseline in plasma $\mathrm{P}$ was apparent following ingestion of diet $\mathrm{E}$, diet $\mathrm{F}$ and diet $\mathrm{G}(1.32,1.50$ and $1.63 \mathrm{mmol} / \mathrm{l}$ and $P=0.023$, $P<0.001$ and $P<0.001$, respectively; Table 3 and Fig. 3(a)). Diets $C$ and D did not show an increase in plasma $P$ and instead were significantly reduced from baseline after $60 \mathrm{~min}(P<0.001$ and $P=0.002$, respectively; Fig. 3(a)), diet $C$ remained decreased until 360 min whilst diet $\mathrm{D}$ did return to baseline at $120 \mathrm{~min}$ before decreasing again for the remainder of the study (Fig. 3(a)). The differences in peak plasma $\mathrm{P}$ and overall kinetic profiles of the diets (Fig. 3(a)) are reflected in the AUC data, where diet $\mathrm{G}$ had the largest AUC compared with all other diets $(0.35(95 \% \mathrm{CI}-0.03,0.73) \mathrm{mmol} / \mathrm{l} \times \mathrm{h}, P \leq 0.0167)$, diets $\mathrm{D}, \mathrm{E}$ and $\mathrm{F}$ were not significantly different to each other, while diets D-G were all significantly higher than diet C $(-1.01$ (95\% CI $-1.41,-0.621) \mathrm{mmol} / \mathrm{l} \times \mathrm{h}, P<0.0216$; Table 3 and Fig. 4). iCa was significantly decreased from baseline for all diets $(P \leq 0.02$; Fig. 3(b)). Diets E, F and G returned to baseline concentrations at $360 \mathrm{~min}$, diet $\mathrm{E}$ also returned to baseline at $240 \mathrm{~min}$ during the study (Fig. 3(b)). At 240 min diet $\mathrm{G}$ was significantly decreased compared with diets $\mathrm{C}(P=0.038)$ and $\mathrm{D}(P=0.047$; Fig. 3(b)). Inorganic $\mathrm{P}$ inclusion also increased peak PTH concentration in a dose-dependent manner. Diets D, E, F and G all significantly increased PTH at 120 min post-meal (fold change 1.33, 1.73, 1.83 and 2.50 , respectively; $P<0.001$, Fig. 3(c)), diet $G$ was still elevated at $240 \mathrm{~min}$ post-meal (fold change $1.47 ; P<0 \cdot 001$, Fig. 3 (c)). Diet C alone showed no observable changes in PTH from baseline throughout the study.

There were no significant differences in $\mathrm{P}$ concentrations in the urine (Table 3).

Study 3 - does the source of added inorganic phosphorus alter postprandial plasma phosphorus kinetics?

Diets including sources of added inorganic P, diets I (SDHP) and $\mathrm{J}$ (sodium tripolyphosphate (STPP), $\mathrm{Na}_{5} \mathrm{P}_{3} \mathrm{O}_{10}$ ), both significantly increased plasma $\mathrm{P}$ to 1.39 and $1.40 \mathrm{mmol} / \mathrm{l}$, respectively, from baseline $(P<0.001$; Fig. 5(a)). Diet H, which did not contain any added inorganic $\mathrm{P}$, showed a significant reduction in plasma $\mathrm{P}$ at $110 \mathrm{~min}(P=0.0139)$ and $150 \mathrm{~min}(P=0.0035)$ before returning to baseline concentration (Fig. 5(a)). There are no significant differences between diet I and diet J for plasma P kinetics (Fig. 5(a)); however, diet I had a significantly higher AUC (0.48 (95\% CI $-0.004,0.82) \mathrm{mmol} / \mathrm{l} \times \mathrm{h})$ than $\operatorname{diet} \mathrm{J}(P=0.0124$; Table 3 and Fig. 6) due to a reduction in plasma $\mathrm{P}$ for diet $\mathrm{J}$ below baseline levels at $190 \mathrm{~min}(P=0.0032), 300 \mathrm{~min}(P=0.0025)$ and $360 \mathrm{~min}(P=0.0472$; Fig. 5(a)). Although iCa was significantly reduced compared with baseline $(P \leq 0.035)$, this response was not influenced by the form of inorganic P (Fig. 5(b)). PTH was elevated above baseline at 110 min post-meal in both diet I and J (1.31- and 1.28-fold increase, respectively; $P<0 \cdot 001$; Fig. 5 (c)) before returning to baseline. Diet $\mathrm{H}$ showed no observable differences in PTH from baseline concentration (Fig. 5(c)).

There were no significant differences in $\mathrm{P}$ concentrations in the urine (Table 3).

Study 4 - when added inorganic phosphorus is fixed, does altering the organic phosphorus content of a diet change postprandial peak plasma phosphorus concentrations?

Diets $\mathrm{K}, \mathrm{L}$ and $\mathrm{M}$ showed a peak in the plasma $\mathrm{P}$ of $1.57,1.50$ and $1.51 \mathrm{mmol} / \mathrm{l}$, respectively (Fig. 7(a)). This peak was unaffected by organic $\mathrm{P}$ content (from raw ingredients) of the diet $(P=1 \cdot 000)$, despite this ranging from $2 \cdot 1$ to $4 \mathrm{~g} / 4184 \mathrm{~kJ}(1000$ kcal). Diet $N$ showed a reduction in plasma $P$ at $240 \mathrm{~min}$ $(P=0.025)$ and at $300 \mathrm{~min}(P=0.005)$ before returning to the baseline concentration (Fig. 7(a)). Diets L, M and $\mathrm{N}$ did not have significantly different AUC ( $P \geq 0 \cdot 178)$; however, diet $\mathrm{K}$ had a significantly higher AUC than the other diets due to the prolonged elevation of plasma P (1.25 (95\% CI 0.629) 1.86) mmol/1 $\times$ h, $P<0.0014$; Table 3$)$. Again iCa was reduced from baseline $(P<0.001)$ with no significant differences detected between diets (Fig. 7(b)). Despite a similar peak in plasma P for diets K, L and M, PTH was found to significantly increase at 110 min only for diets $\mathrm{L}$ and $\mathrm{M}(8.89$ and $10.60 \mathrm{pg} / \mathrm{ml}$, respectively; Fig. 7(c)). PTH concentrations following ingestion of diet $M$ were significantly higher than that with diet $\mathrm{N}$ at $110 \mathrm{~min}(P=0.003)$ and diet $\mathrm{K}$ was significantly higher than diet $\mathrm{L}$ at $360 \mathrm{~min}(P=0 \cdot 004$; Fig. 7(c)). Diet $\mathrm{N}$ showed no observable differences in PTH from baseline concentration.

There were no significant differences in $\mathrm{P}$ concentrations in the urine (Table 3). 
Table 3. Data for each diet specificationt

(Mean values and $95 \%$ confidence intervals)

\begin{tabular}{|c|c|c|c|c|c|c|c|c|c|c|c|c|c|c|c|c|}
\hline \multirow[b]{2}{*}{$\begin{array}{l}\text { Diet specifications } \\
(\mathrm{g} / 4184 \mathrm{~kJ}(1000 \mathrm{kcal}))\end{array}$} & \multicolumn{2}{|c|}{$\begin{array}{l}\text { Time to peak plasma: } \\
\text { mean peak plasma } \mathrm{P}(\mathrm{mmol} / \mathrm{l})\end{array}$} & \multicolumn{2}{|c|}{$\begin{array}{l}\text { Mean P AUC } \\
(\mathrm{mmol} / \mathrm{l} \times \mathrm{h})\end{array}$} & \multicolumn{2}{|c|}{$\begin{array}{l}\text { Ionised } \mathrm{Ca} \text { area between } \\
\text { baseline and curve } \\
(\mathrm{mmol} / \mathrm{x} \times \mathrm{h})\end{array}$} & \multicolumn{2}{|c|}{ Peak PTH (pg/ml) } & \multicolumn{2}{|c|}{$\begin{array}{l}\text { Mean urine P excretion } \\
(\mathrm{mg} / \mathrm{kg} \mathrm{BW})\end{array}$} & \multicolumn{2}{|c|}{$\begin{array}{l}\text { Mean } P \text { intake over sample } \\
\text { day }(\mathrm{mg} / \mathrm{kg} \mathrm{BW})\end{array}$} & \multicolumn{2}{|c|}{$\begin{array}{l}\text { Test diet P intake } \\
(\mathrm{mg} / \mathrm{kg} \mathrm{BW})\end{array}$} & \multicolumn{2}{|c|}{$\begin{array}{l}\text { Test diet added P intake } \\
(\mathrm{mg} / \mathrm{kg} \mathrm{BW})\end{array}$} \\
\hline & Mean & $95 \% \mathrm{Cl}$ & Mean & $95 \% \mathrm{Cl}$ & Mean & $95 \% \mathrm{Cl}$ & Mean & $95 \% \mathrm{Cl}$ & Mean & $95 \% \mathrm{Cl}$ & Mean & $95 \% \mathrm{Cl}$ & Mean & $95 \% \mathrm{Cl}$ & Mean & $95 \% \mathrm{Cl}$ \\
\hline $\begin{array}{l}\text { Diet A: total P } 4.78 \mathrm{~g}, 3.5 \mathrm{~g} \text { from } \\
\text { SDHP, Ca:P } 0.59\end{array}$ & $150 \mathrm{~min}^{*}: 1.976$ & $150 \mathrm{~min}: 1 \cdot 724,2 \cdot 266$ & $3.65^{\mathrm{a}}$ & $2 \cdot 80,4 \cdot 49$ & $-0.337^{\mathrm{a}}$ & $-0.495,-0.178$ & $31.83^{\mathrm{a}}$ & $24 \cdot 32,41 \cdot 67$ & $56 \cdot 6^{\mathrm{a}}$ & $45 \cdot 1,68 \cdot 2$ & $161 \cdot 24$ & $153 \cdot 09,169 \cdot 39$ & $100 \cdot 98$ & $86 \cdot 67,117.62$ & 72.63 & $63 \cdot 43,83 \cdot 15$ \\
\hline $\begin{array}{l}\text { Diet B: total P } 3.38 \mathrm{~g} \text {, no added } \\
\text { inorganic } \mathrm{P}, \mathrm{Ca}: \mathrm{P}_{1.55}\end{array}$ & $\begin{array}{l}0 \min \text { (no peak): } \\
1 \cdot 211\end{array}$ & $\begin{array}{l}0 \min (\text { no peak): } \\
1.053,1.392\end{array}$ & $-1 \cdot 1^{b}$ & $-1.4,-0.621$ & $-0.145^{\mathrm{b}}$ & $-0.285,-0.00553$ & $8.07^{\mathrm{b}}$ & $6 \cdot 11,10 \cdot 65$ & $29 \cdot 2^{\mathrm{b}}$ & $17 \cdot 4,41 \cdot 0$ & 130.80 & $123.72,137.87$ & 68.37 & $58.64,79.69$ & 0 & \\
\hline $\begin{array}{l}\text { Diet C: total P } 1.13 \mathrm{~g} \text {, no added } \\
\text { inorganic P Ca:P } 1.23\end{array}$ & 0 min (no peak): & $0 \min _{1 \cdot 14.1 \cdot 35}$ (no peak): & $-1.01^{\mathrm{a}}$ & $-1 \cdot 4,-0.621$ & $-0.26^{\mathrm{a}}$ & $-0.347,-0.174$ & $9 \cdot 17^{\mathrm{a}}$ & $7 \cdot 58,11 \cdot 1$ & $28 \cdot 4^{\mathrm{a}}$ & $21 \cdot 0,35 \cdot 9$ & 89.21 & $80 \cdot 67,97 \cdot 75$ & 25.56 & $22 \cdot 80,28 \cdot 64$ & 0 & \\
\hline $\begin{array}{l}\text { Diet D: total P } 4.02,0.5 \mathrm{~g} \text { from } \\
\text { STPP, Ca:P } 1.63\end{array}$ & 120 min* $^{*}: 1 \cdot 26$ & $120 \mathrm{~min}: 1 \cdot 16,1 \cdot 37$ & $-0.516^{b}$ & $-0.896,-0.136$ & $-0.278^{a}$ & $-0.368,-0.189$ & $11 \cdot 6^{\mathrm{b}}$ & $9 \cdot 6,14 \cdot 0$ & $30 \cdot 2^{\mathrm{a}}$ & $23 \cdot 3,37 \cdot 1$ & 150.48 & $141.94,159.02$ & 87.29 & $78 \cdot 12,97.52$ & $22 \cdot 42$ & $20 \cdot 26,24 \cdot 81$ \\
\hline $\begin{array}{l}\text { Diet E: total P } 4.48 \mathrm{~g}, 0.75 \mathrm{~g} \text { from } \\
\text { STTP, Ca:P } 1.59\end{array}$ & $120 \mathrm{~min}^{*}: 1 \cdot 32$ & $120 \mathrm{~min}: 1 \cdot 21,1 \cdot 44$ & $-0.197^{b}$ & $-0.585,0 \cdot 191$ & $-0.26^{\mathrm{a}}$ & $-0.35,-0.17$ & $14 \cdot 0^{\mathrm{b}, \mathrm{c}}$ & $11 \cdot 5,17 \cdot 0$ & $35 \cdot 2^{a}$ & $28 \cdot 0,42 \cdot 3$ & 161.09 & $152.55,169.64$ & 94.05 & $84.18,105.07$ & 73.67 & $30 \cdot 47,37 \cdot 20$ \\
\hline $\begin{array}{l}\text { Diet F: total P } 4.48 \mathrm{~g}, 1 \mathrm{~g} \text { from } \\
\text { STPP, Ca:P } 1.48\end{array}$ & $120 \mathrm{~min}^{*}: 1.5$ & $120 \mathrm{~min}: 1 \cdot 38,1.63$ & $-0.151^{b}$ & $-0.539,0.236$ & $-0.253^{a}$ & $-0.336,-0.17$ & $16 \cdot 0^{\mathrm{c}, \mathrm{d}}$ & $13 \cdot 2,19 \cdot 3$ & $32 \cdot 2^{\mathrm{a}}$ & $25 \cdot 0,39 \cdot 4$ & 159.28 & $150 \cdot 73,167 \cdot 82$ & $96 \cdot 68$ & $86.54,108.01$ & $45 \cdot 19$ & $40 \cdot 92,49.88$ \\
\hline $\begin{array}{l}\text { Diet G: total P } 4.9 \mathrm{~g}, 1.5 \mathrm{~g} \text { from } \\
\text { STPP, Ca:P } 1.64\end{array}$ & $120 \mathrm{~min}^{*}: 1.63$ & $120 \mathrm{~min}: 1 \cdot 50,1 \cdot 78$ & $0.35^{c}$ & $-0.0305,0.731$ & $-0.344^{a}$ & $-0.448,-0.241$ & $20 \cdot 4^{\mathrm{e}}$ & $16 \cdot 9,24 \cdot 6$ & $37 \cdot 3^{\mathrm{a}}$ & $29 \cdot 8,44 \cdot 7$ & 168.49 & $159.95,177.03$ & $102 \cdot 25$ & $91 \cdot 53,114 \cdot 22$ & 67.05 & $60 \cdot 77,73 \cdot 98$ \\
\hline $\begin{array}{l}\text { Diet } \mathrm{H} \text { : total } \mathrm{P} 3.79 \mathrm{~g} \text {, no added } \\
\text { inorganic } \mathrm{P}, \mathrm{Ca}: \mathrm{P} 1.6\end{array}$ & $360 \mathrm{~min}: 1.24$ & $360 \mathrm{~min}: 1 \cdot 13,1 \cdot 36$ & $-0.277^{\mathrm{a}}$ & $-0.688,0.134$ & $-0.0963^{a}$ & $-0.157,-0.0351$ & $7 \cdot 46^{\mathrm{a}}$ & $6.13,9.07$ & $26 \cdot 58^{\mathrm{a}}$ & $20.54,32 \cdot 62$ & 138.99 & $130 \cdot 62,147 \cdot 36$ & 77.06 & $69 \cdot 32,85 \cdot 64$ & 0 & \\
\hline $\begin{array}{l}\text { Diet I: total P } 3.86 \mathrm{~g}, 1.5 \mathrm{~g} \text { from } \\
\text { SDHP, Ca:P } 1.59\end{array}$ & $110 \min ^{*}: 1 \cdot 40$ & $110 \mathrm{~min}: 1 \cdot 28,1 \cdot 54$ & $0.48^{b}$ & $-0.00355,0.82$ & $-0.165^{a}$ & $-0.226,-0.104$ & $9 \cdot 18^{\mathrm{b}}$ & $7 \cdot 57,11 \cdot 13$ & $27 \cdot 76^{\mathrm{a}}$ & $21 \cdot 69,33 \cdot 83$ & $136 \cdot 23$ & $126 \cdot 50,145 \cdot 97$ & 74.64 & $67 \cdot 15,82 \cdot 96$ & 29.20 & $26 \cdot 54,32 \cdot 12$ \\
\hline $\begin{array}{l}\text { Diet J: total P } 3.52 \mathrm{~g}, 1.5 \mathrm{~g} \text { from } \\
\text { STPP, Ca:P } 1.7\end{array}$ & $60 \mathrm{~min}^{*}: 1 \cdot 40$ & $60 \min : 1.28,1.53$ & $-0.146^{\mathrm{a}}$ & $-0.558,0.265$ & $-0.198^{b}$ & $-0.259,-0.137$ & $9 \cdot 07^{b}$ & $7 \cdot 45,11 \cdot 03$ & $25 \cdot 36^{\mathrm{a}}$ & $19 \cdot 32,31 \cdot 40$ & 132.67 & $123 \cdot 64,141 \cdot 70$ & 70.87 & $63 \cdot 75,78 \cdot 78$ & 30.12 & $27 \cdot 38,33 \cdot 13$ \\
\hline $\begin{array}{l}\text { Diet K: total P } 3.59,1.5 \mathrm{~g} \text { from } \\
\text { SDHP, Ca:P } 0.93\end{array}$ & $360 \min ^{*}: 1.57$ & 360 min: $1.44,1 \cdot 70$ & $1 \cdot 25^{\mathrm{a}}$ & $0.629,1.86$ & $-0.24^{a}$ & $-0.31,-0.169$ & $7 \cdot 81^{\mathrm{a}, \mathrm{b}}$ & $6 \cdot 00,10 \cdot 20$ & $38.1^{a}$ & $28 \cdot 7,47 \cdot 6$ & 149.76 & $139 \cdot 40,159 \cdot 82$ & 86.74 & $75 \cdot 85,99 \cdot 18$ & 33.98 & $30 \cdot 11,38 \cdot 33$ \\
\hline $\begin{array}{l}\text { Diet L: total P } 4.17 \mathrm{~g}, 1.5 \mathrm{~g} \text { from } \\
\text { STPP Ca:P } 1.88\end{array}$ & $110 \mathrm{~min}^{*}: 1.5$ & $110 \mathrm{~min}: 1 \cdot 36,1 \cdot 64$ & $-0.241^{b}$ & $-0.882,0.400$ & $-0.265^{a}$ & $-0.339,-0.192$ & $8.89^{\mathrm{a}, \mathrm{b}}$ & $6 \cdot 77,11 \cdot 70$ & $31 \cdot 0^{\mathrm{a}}$ & $21 \cdot 0,41 \cdot 0$ & 155.63 & $139 \cdot 16,172 \cdot 09$ & 91.00 & $79 \cdot 14,104 \cdot 61$ & 32.81 & $28.93,37 \cdot 20$ \\
\hline $\begin{array}{l}\text { Diet M: total P } 5.5 \mathrm{~g}, 1.5 \mathrm{~g} \text { from } \\
\text { STPP, Ca:P } 1.7\end{array}$ & $110 \min ^{*}: 1.51$ & $110 \mathrm{~min}: 1.37,1.65$ & $0.0868^{\mathrm{b}}$ & $-0.554,0.728$ & $-0.231^{b}$ & $-0.304,-0.158$ & $10 \cdot 60^{\mathrm{a}}$ & $8.09,14.00$ & $40 \cdot 9^{\mathrm{a}}$ & $29 \cdot 4,52 \cdot 4$ & 184.09 & $166 \cdot 78,201 \cdot 41$ & 119.79 & $104 \cdot 22,137 \cdot 66$ & 32.07 & $28 \cdot 27,36 \cdot 35$ \\
\hline $\begin{array}{l}\text { Diet N: total P } 4.85 \mathrm{~g}, 0.5 \mathrm{~g} \text { from } \\
\text { STPP, Ca:P } 1.47\end{array}$ & 0 min (no peak): & $0 \min _{1 \cdot 17,1 \cdot 45}$ (no peak): & $-0.577^{b}$ & $-1.24,0.0895$ & $-0.238^{a}$ & $-0.315,-0.162$ & $7 \cdot 23^{\mathrm{b}}$ & $5.41,9.67$ & $33.5^{\mathrm{a}}$ & $22 \cdot 8,44 \cdot 1$ & $151 \cdot 14$ & $129.97,172 \cdot 30$ & 83.43 & $72 \cdot 10,96 \cdot 53$ & 8.71 & $7.55,10.03$ \\
\hline
\end{tabular}

PTH, parathyroid hormone; BW, body weight; SDHP, sodium dihydrogen phosphate; STPP, sodium tripolyphosphate.

a,b,c,d,e Mean values with unlike superscript letters were significantly different between diets within each study $(P<0.05)$.

* Time to peak plasma shows significant difference to baseline results.

† Separate trials are delineated by a blank line. 

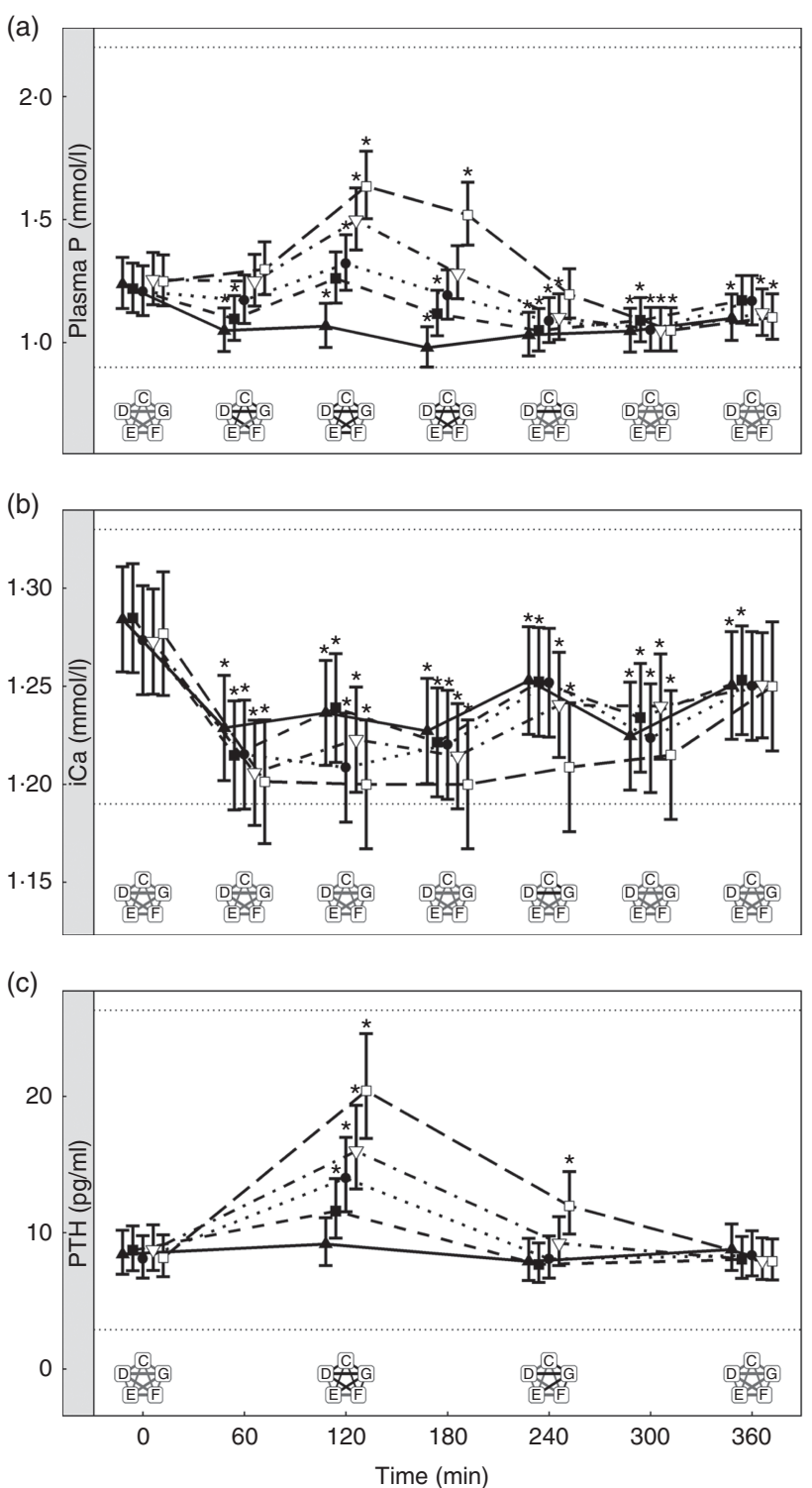

Fig. 3. Biochemical parameters over the study day for study 2. (a) Plasma phosphorus, (b) ionised calcium (iCa) and (c) parathyroid hormone (PTH). Diet $C(\leftarrow): 1.13 \mathrm{~g}$ total phosphorus/4184 kJ (1000 kcal), no added inorganic phosphorus, calcium:phosphorus 1.23. Diet $D\left(-{ }^{-}\right.$): $4.02 \mathrm{~g}$ total phosphorus $/ 4184 \mathrm{~kJ}(1000 \mathrm{kcal}), \quad 0.5 \mathrm{~g}$ phosphorus from sodium tripolyphosphate (STPP)/4184 kJ (1000 kcal), calcium:phosphorus 1.63. Diet E (. O . ): $4.48 \mathrm{~g}$ total phosphorus $/ 4184 \mathrm{~kJ}(1000 \mathrm{kcal}), 0.75 \mathrm{~g}$ phosphorus from STPP/4184 kJ (1000 kcal), calcium:phosphorus 1.55. Diet F (.- $\nabla-.): 4.45 \mathrm{~g}$ total phosphorus $/ 4184 \mathrm{~kJ}(1000 \mathrm{kcal}), 1.0 \mathrm{~g}$ phosphorus from STPP/4184 kJ (1000 kcal), calcium:phosphorus 1.48 . Diet $\mathrm{G}(-\square-): 4.9 \mathrm{~g}$ total phosphorus/ $4184 \mathrm{~kJ}$ (1000 kcal), $1.5 \mathrm{~g}$ phosphorus from STPP/4184 kJ (1000 kcal), calcium:phosphorus 1.64 . Values are means, with $95 \% \mathrm{Cl}$ represented by vertical bars. * Mean values were significantly different from baseline $(P<0.05)$. Reference ranges are shown for each parameter. Significant differences between diets: false $(\square)$ and true $(\square)$.

\section{Does the dietary calcium:phosphorus ratio influence postprandial plasma phosphorus kinetics?}

To enable this comparison, data from the separate studies have been combined. While these data cannot be statistically compared, visual inspection of the kinetic profiles shows that SDHPsupplemented diets A (Ca:P 0.56) and K (Ca:P 0.93), both with $\mathrm{Ca}: \mathrm{P}$ below $1 \cdot 0$, displayed a prolonged elevation of postprandial

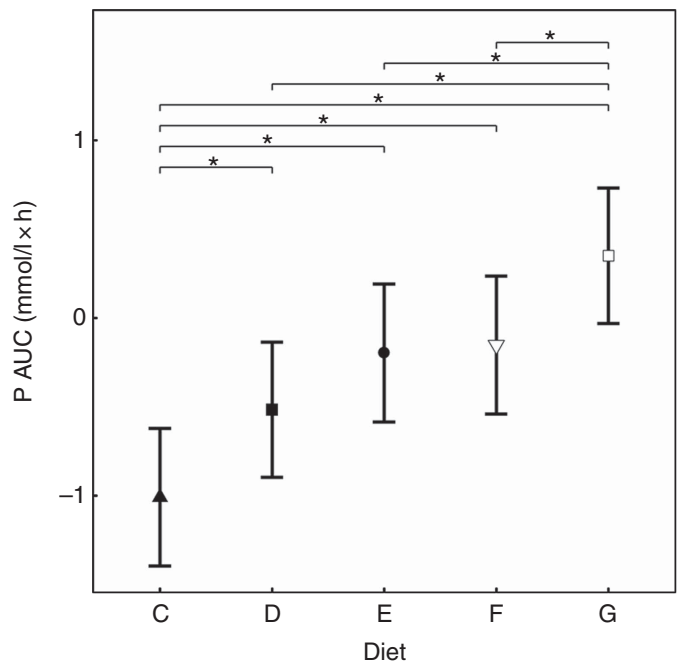

Fig. 4. AUC data for study 2. Diet $C(\mathbf{A}): 1.13 \mathrm{~g}$ total phosphorus/4184 $\mathrm{kJ}$ (1000 kcal), $100 \%$ organic phosphorus, calcium:phosphorus 1.23. Diet D ( $\square$ ): $4.02 \mathrm{~g}$ total phosphorus $/ 4184 \mathrm{~kJ}(1000 \mathrm{kcal}), 0.5 \mathrm{~g}$ phosphorus from sodium tripolyphosphate (STPP)/4184 kJ (1000 kcal), calcium:phosphorus 1.63. Diet $\mathrm{E}$ (O): $4.48 \mathrm{~g}$ total phosphorus $/ 4184 \mathrm{~kJ}(1000 \mathrm{kcal}), 0.75 \mathrm{~g}$ phosphorus from STPP/4184 kJ (1000 kcal), calcium:phosphorus 1.55. Diet $F(\nabla): 4.45 \mathrm{~g}$ total phosphorus $/ 4184 \mathrm{~kJ}$ (1000 kcal), $1.0 \mathrm{~g}$ phosphorus from STPP/4184 kJ (1000 kcal), calcium:phosphorus 1.48. Diet $\mathrm{G}(\square): 4.9 \mathrm{~g}$ total phosphorus $/ 4184 \mathrm{~kJ}$ (1000 kcal), $1.5 \mathrm{~g}$ phosphorus from STPP $/ 4184 \mathrm{~kJ}$ (1000 kcal), calcium:phosphorus 1.64. Values are means, with $95 \% \mathrm{Cl}$ represented by vertical bars. ${ }^{*}$ Mean values were significantly different from baseline $(P<0.05)$.

plasma P compared with diet I (Ca:P 1.59) where the Ca:P was in excess of 1.0 (Fig. 9(a)). These differences in plasma $\mathrm{P}$ kinetic profiles resulted in a marked increase in the AUC for diet A (3.65 (95\% CI 2.8, 4.49) $\mathrm{mmol} / \mathrm{l} \times \mathrm{h})$ and diet $\mathrm{K}(1.25(95 \% \mathrm{CI}$ $0.629,1.86) \mathrm{mmol} / \mathrm{l} \times \mathrm{h})$ compared with diet I (0.48 (95\% CI $-0.004,0.82) \mathrm{mmol} / \mathrm{l} \times \mathrm{h}$; Fig. 10 and Table 3). In addition, changes in iCa (Fig. 9(b)), PTH (Fig. 9(c)) and urinary P excretion (Table 3) were most evident when diet A was fed, which contained the highest P from SDHP of $3.5 \mathrm{~g} / 4184 \mathrm{~kJ}$ (1000 kcal) and lowest Ca:P ratio of $0 \cdot 56$, whereas PTH did not change from baseline following ingestion of diets K and I (Fig. 9 (c)), which contained $1.5 \mathrm{~g}$ P from SDHP/4184 kJ (1000 kcal) and higher Ca:P ratios of 0.93 and 1.59 , respectively.

\section{Discussion}

In an effort to better understand the influence of dietary $\mathrm{P}$ sources and levels on postprandial biochemical parameters in healthy neutered cats, a series of studies were undertaken to evaluate plasma P, iCa and PTH kinetic responses. The data presented here demonstrate that added inorganic $\mathrm{P}$ sources SDHP and STPP cause a greater, dose-dependent peak in plasma $P$ than natural sources.

An initial proof of principle study was performed to assess whether two distinct diets, with regard to total $\mathrm{P}$, inorganic $\mathrm{P}$ inclusion and $\mathrm{Ca}: \mathrm{P}$, resulted in marked differences in postprandial plasma $\mathrm{P}$ kinetics. The high plasma $\mathrm{P}$ concentrations observed with diet $\mathrm{A}$ indicate that $\mathrm{P}$ is absorbed faster than regulating factors can respond to maintain stable plasma P (Fig. 1(a)). This was accompanied by decreased plasma iCa concentrations and an elevation in plasma PTH, which apparently resulted in 

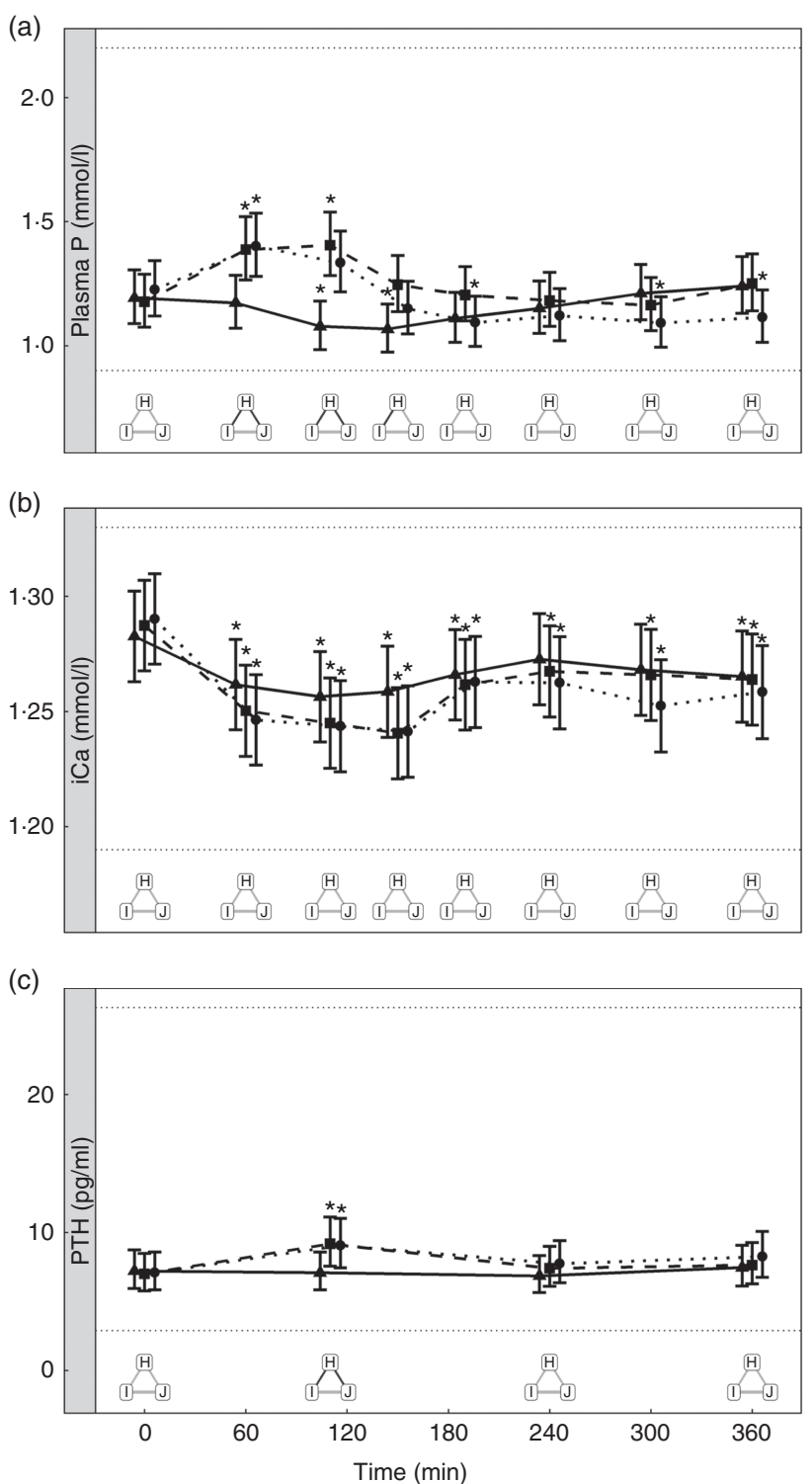

Fig. 5. Biochemical parameters over the study day for study 3. (a) Plasma phosphorus, (b) ionised calcium (iCa) and (c) parathyroid hormone (PTH). Diet $\mathrm{H}(\longrightarrow-): 3.79 \mathrm{~g}$ total phosphorus $/ 4184 \mathrm{~kJ}$ (1000 kcal), no added inorganic phosphorus, calcium:phosphorus 1.6. Diet I (- .): $3.86 \mathrm{~g}$ total phosphorus/ $4184 \mathrm{~kJ}$ (1000 kcal), $1.5 \mathrm{~g}$ phosphorus from sodium dihydrogen phosphate/ $4184 \mathrm{~kJ}(1000 \mathrm{kcal})$, calcium:phosphorus 1.59. Diet $\mathrm{J}(\cdot \cdot \cdots): 3.52 \mathrm{~g}$ total phosphorus $/ 4184 \mathrm{~kJ}(1000 \mathrm{kcal}), 1.5 \mathrm{~g}$ phosphorus from sodium tripolyphosphate/4184 kJ (1000 kcal), calcium:phosphorus 1.7. Values are means, with $95 \% \mathrm{Cl}$ represented by vertical bars. * Mean values were significantly different from baseline $(P<0.05)$. Reference ranges are shown for each parameter. Significant differences between diets: false $(\square)$ and true $(\square)$.

higher urinary $\mathrm{P}$ excretion. This is consistent with other studies investigating $\mathrm{P}$ homoeostasis ${ }^{(22,37)}$. In addition, Finco et al. $^{(8)}$ reported that a diet enriched with inorganic $\mathrm{P}$ showed a higher percentage of dietary $\mathrm{P}$ recovered in the urine than a fully organic $\mathrm{P}$ diet (34.9\% compared with $14.7 \%$ ). While PTH release stimulates bone resorption and limits bone formation in humans ${ }^{(38,39)}$, in rats and hamsters radiolabelled $\mathrm{P}$ was found in femur heads $4 \mathrm{~h}$ after ingestion ${ }^{(40)}$. This PTH-driven bone resorption may have been apparent in diet A from the proof of concept study, where there was a sustained change in plasma P and PTH apparent, and data

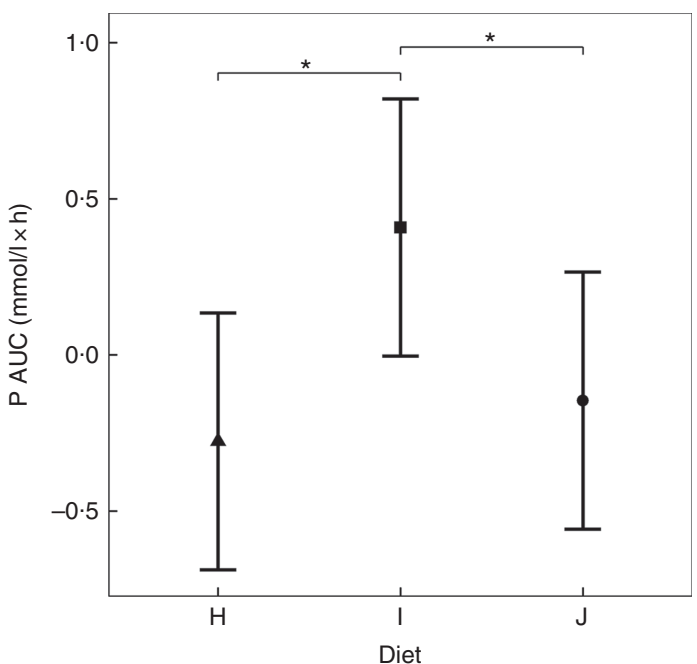

Fig. 6. AUC data for study 3. Diet $\mathrm{H}(\mathbf{\Delta}): 3.79 \mathrm{~g}$ total phosphorus $/ 4184 \mathrm{~kJ}$ (1000 kcal), no added inorganic phosphorus, calcium:phosphorus 1.6. Diet I ): $3.86 \mathrm{~g}$ total phosphorus $/ 4184 \mathrm{~kJ}(1000 \mathrm{kcal}), 1.5 \mathrm{~g}$ phosphorus from sodium dihydrogen phosphate/4184 kJ (1000 kcal), calcium:phosphorus 1.59 . Diet J (O): $3.52 \mathrm{~g}$ total phosphorus $/ 4184 \mathrm{~kJ}$ (1000 kcal), $1.5 \mathrm{~g}$ phosphorus from sodium tripolyphosphate/4184 kJ (1000 kcal), calcium:phosphorus 1.7 . Values are means, with $95 \% \mathrm{Cl}$ represented by vertical bars. * Mean values were significantly different from baseline $(P<0.05)$.

from feeding this diet over 4 weeks showed increased circulating concentrations of bone-specific alkaline phosphatase (BAP) and C-terminal cross-linking telopeptide of type I collagen $(\mathrm{CTX})^{(20)}$. This may be the case for the other diets tested here, but bone turnover markers were not measured in the present study, and the urine data may not be sufficiently sensitive to detect subtle differences between diets in this model. Interestingly, diet K, despite causing a prolonged elevation in plasma $\mathrm{P}$, showed no discernible peak in postprandial PTH, as well as no measurable changes in BAP and CTX when fed during a 30 -week feeding trial ${ }^{(20)}$. We cannot say for certain that PTH does not have a peak before the $2 \mathrm{~h}$ timepoint (as this was not quantified); however, due to the increasing PTH concentration in the pilot study it would seem unlikely. FGF-23 has also been implicated in the homoeostatic control of plasma P concentrations, where FGF-23 release is stimulated in response to high dietary $\mathrm{P}$ consumption. This is thought to increase renal $\mathrm{P}$ excretion and reduce 1,25-dihydroxyvitamin $\mathrm{D}_{3}$ production by the kidney in order to reduce intestinal absorption of $\mathrm{P}^{(11)}$. Significant postprandial changes in FGF-23 were not observed during study 1 (Fig. 1(d)). Although FGF-23 was analysed using a human antibody-based assay, it has been validated for use in cats ${ }^{(31)}$ and has been used several times by the same research group to show changes in FGF-23 in cats with $\mathrm{CKD}^{(41-44)}$. In Alexander et $a l .{ }^{(20)}$ blinded samples were tested and a difference in FGF-23 was found between control and test groups. These data taken together suggest the suitability of the assay for cats, despite the target species for the assay being human. Consequently FGF-23 was not measured beyond the initial proof of principle study. Acute phosphate loading in healthy men has also been shown to have little effect on serum FGF-23 concentrations ${ }^{(45)}$, suggesting that short-term $\mathrm{P}$ exposure may not be sufficient to illicit a response. Isakova et al. ${ }^{(11)}$ suggested that FGF-23 may not be an acute regulator of $\mathrm{P}$ handling as well as the potential that the $\mathrm{P}$ loading in 

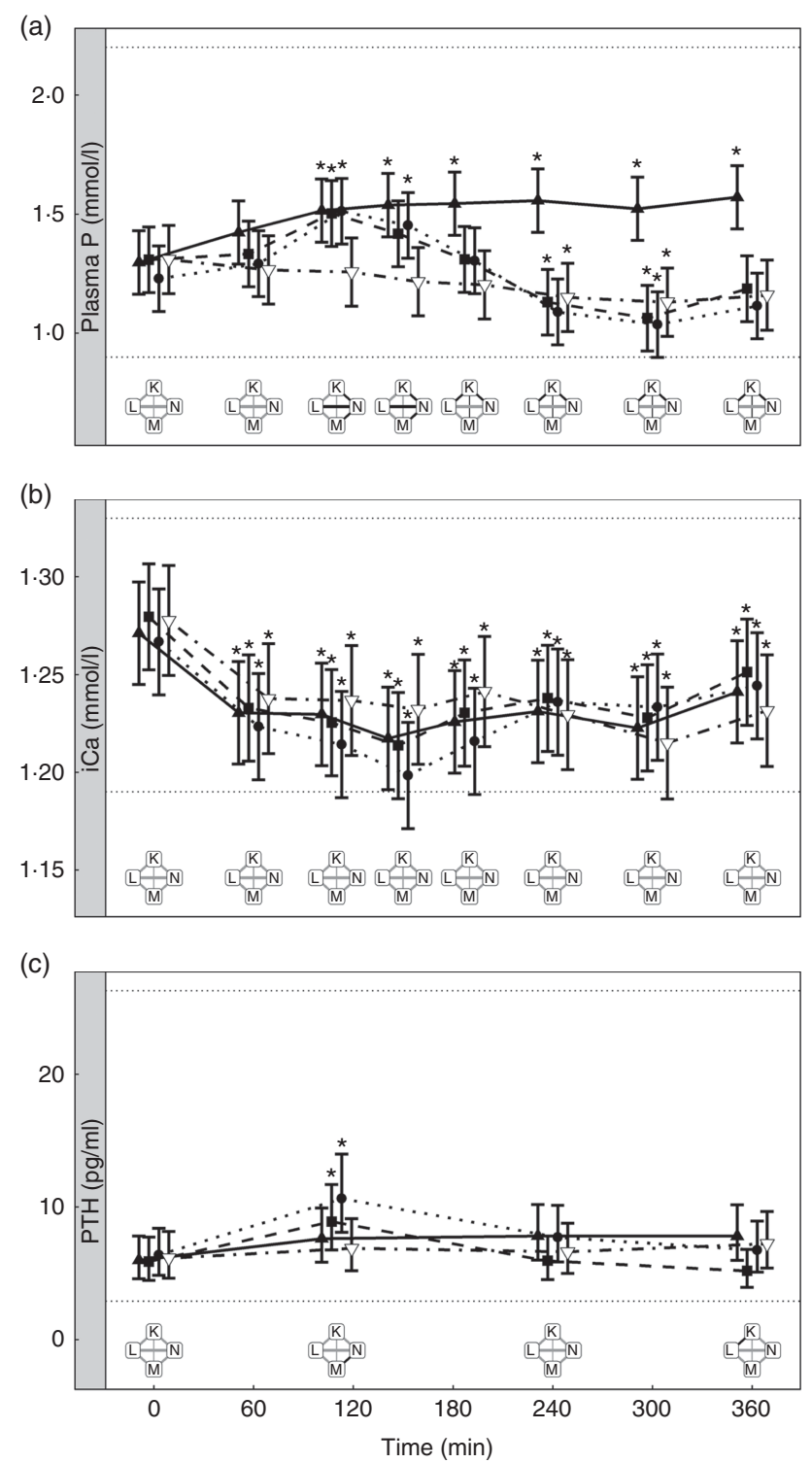

Fig. 7. Biochemical parameters over the study day for study 4. (a) Plasma phosphorus, (b) ionised calcium (iCa) and (c) parathyroid hormone (PTH). Diet $\mathrm{K}(\multimap-$ ): $3.59 \mathrm{~g}$ total phosphorus $/ 4184 \mathrm{~kJ}$ (1000 kcal), $1.5 \mathrm{~g}$ phosphorus from sodium dihydrogen phosphate $/ 4184 \mathrm{~kJ}(1000 \mathrm{kcal})$, calcium:phosphorus 0.93. Diet L (- . :) $4.17 \mathrm{~g}$ total phosphorus $/ 4184 \mathrm{~kJ}$ (1000 kcal), $1.5 \mathrm{~g}$ phosphorus from sodium tripolyphosphate (STPP)/4184 kJ (1000 kcal), calcium:phosphorus 1.88. Diet M (. - . ) $5.5 \mathrm{~g}$ total phosphorus $/ 4184 \mathrm{~kJ}$ (1000 kcal), $1.5 \mathrm{~g}$ phosphorus from STPP/4184 kJ (1000 kcal), calcium:phosphorus 1.7. Diet $\mathrm{N}(\cdot-\nabla-\cdot): 4.85 \mathrm{~g}$ total phosphorus $/ 4184 \mathrm{~kJ}(1000 \mathrm{kcal}), 0.5 \mathrm{~g}$ phosphorus from STPP/4184 kJ (1000 kcal), calcium:phosphorus 1.47. Values are means, with $95 \% \mathrm{Cl}$ represented by vertical bars. * Mean values were significantly different from baseline $(P<0.05)$. Reference ranges are shown for each parameter. Significant differences between diets: false $(\square)$ and true $(\square)$.

their study was not sufficiently high enough to elicit a response ${ }^{(11)}$. This latter point seems plausible as there is a study in rats showing that FGF-23 was raised $3 \mathrm{~h}$ post-ingestion by a $90 \mathrm{mg} \mathrm{P} / \mathrm{kg}$ supplement ${ }^{(46)}$, which is higher than the $72.63 \mathrm{mg} / \mathrm{kg} \mathrm{BW}$ fed in diet A here. In the first study, increased plasma $\mathrm{P}$ was associated with an increase in $24 \mathrm{~h}$ urinary $\mathrm{P}$ excretion, which is consistent with observations in healthy female volunteers offered inorganic phosphate supplements ${ }^{(25,38)}$. When comparing this data with other cat

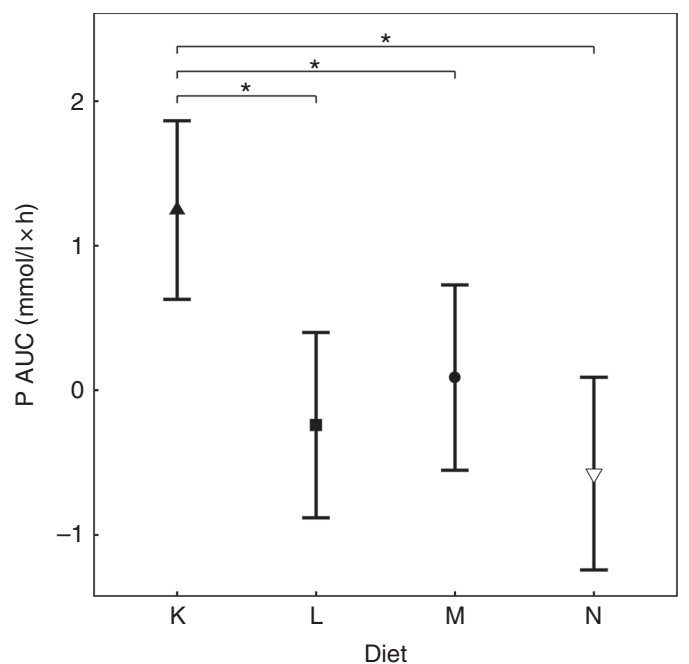

Fig. 8. AUC data for study 4. Diet $\mathrm{K}(\mathbf{\Delta}): 3.59 \mathrm{~g}$ total phosphorus $/ 4184 \mathrm{~kJ}$ (1000 kcal), $1.5 \mathrm{~g}$ phosphorus from sodium dihydrogen phosphate $/ 4184 \mathrm{~kJ}$ (1000 kcal), calcium:phosphorus 0.93. Diet $L(\square): 4.17 \mathrm{~g}$ total phosphorus/ $4184 \mathrm{~kJ}$ (1000 kcal), $1.5 \mathrm{~g}$ phosphorus from sodium tripolyphosphate (STPP)/ $4184 \mathrm{~kJ}$ (1000 kcal), calcium:phosphorus 1.88. Diet $\mathrm{M}(\bigcirc): 5.5 \mathrm{~g}$ total phosphorus $/ 4184 \mathrm{~kJ}$ (1000 kcal), $1.5 \mathrm{~g}$ phosphorus from STPP/4184 kJ (1000 kcal), calcium:phosphorus 1.7. Diet $\mathrm{N}(\nabla): 4.85 \mathrm{~g}$ total phosphorus $/ 4184 \mathrm{~kJ}$ (1000 kcal), $0.5 \mathrm{~g}$ phosphorus from STPP/4184 kJ (1000 kcal), calcium:phosphorus 1.47. Values are means, with $95 \% \mathrm{Cl}$ represented by vertical bars. * Mean values were significantly different from baseline $(P<0.05)$.

studies, feeding a purified diet containing 3.3 g P from SDHP/4184 $\mathrm{kJ}$ (1000 kcal) for $21 \mathrm{~d}$ before a $7 \mathrm{~d}$ urine collection showed a renal excretion of almost $80 \%$ (219.02 mg P/kg body weight (BW) per $\mathrm{d}$ excreted $)^{(3)}$. Finco et al. ${ }^{(8)}$ collected urine over $6 \mathrm{~d}$ from cats fed a diet containing $2 \cdot 1 \mathrm{~g}$ P from SDHP/4184 kJ (1000 kcal) and found a renal excretion of $35 \%(70.8 \mathrm{mg} \mathrm{P} / \mathrm{kg} \mathrm{BW}$ per d excreted), which agrees with our $35.1 \%$ (56.6 mg P/kg BW per d). The disparity between the pilot study data here and the data containing a similar amount of SDHP may be due to the purified diet, by definition this diet contains more readily available ingredients and thus will be more easily absorbed.

To further understand the dietary factors responsible for the marked increase in plasma $\mathrm{P}$ observed in study 1 , a series of follow-up studies were undertaken to determine the effect of added inorganic $\mathrm{P}$, source of $\mathrm{P}$ and increasing total $\mathrm{P}$ using organic sources of $\mathrm{P}$ on postprandial plasma $\mathrm{P}$ kinetics. In interrogating these data, an observation related to Ca:P was also noted and will be discussed as a factor responsible for differences in the regulation of postprandial $\mathrm{P}$.

When dietary inorganic $\mathrm{P}$ inclusion was increased from 0 to $1.5 \mathrm{~g} / 4184 \mathrm{~kJ}$ (1000 kcal) while maintaining a fixed Ca:P, plasma $\mathrm{P}$ was elevated in a dose-dependent manner (Fig. 3(a)). At least under the conditions applied in the present studies, added inorganic $\mathrm{P}$ appears primarily responsible for driving the increase in postprandial plasma $\mathrm{P}$. In addition, the present data demonstrate that despite increasing the organic $\mathrm{P}$ inclusion from $2 \cdot 1$ to $4 \mathrm{~g} / 4184 \mathrm{~kJ}(1000 \mathrm{kcal})$, while maintaining a fixed added inorganic P of $1.5 \mathrm{~g} / 4184 \mathrm{~kJ}(1000 \mathrm{kcal})$ and $\mathrm{Ca}: \mathrm{P}>1 \cdot 5$, there was no difference in the peak plasma $\mathrm{P}$ concentrations (Fig. 7(a)) or plasma P AUC (Fig. 8) apparent between the diets. While this supports other studies reporting that inorganic sources of $\mathrm{P}$ are readily digested and highly bioavailable (80-100\%) 

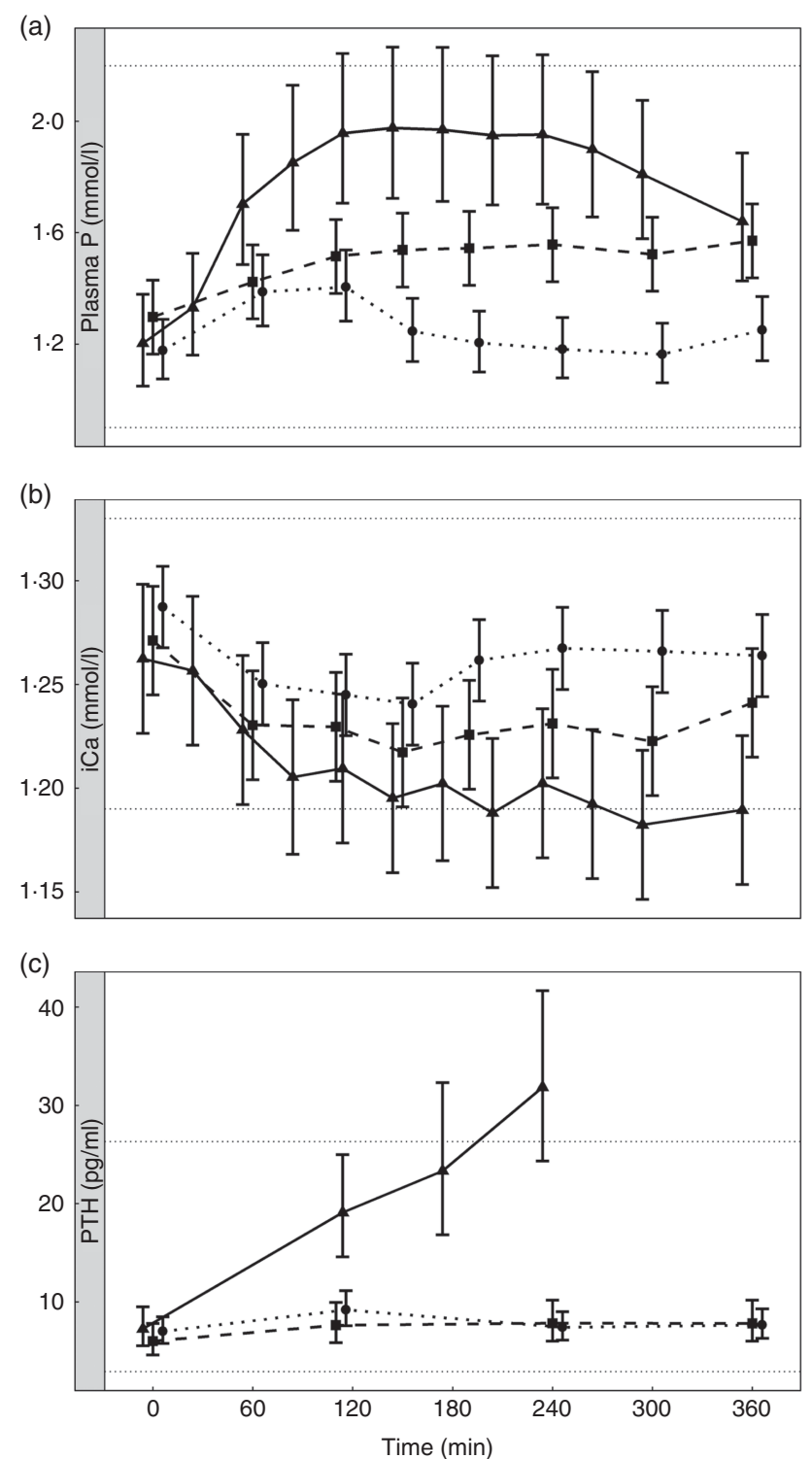

Fig. 9. Postprandial biochemical parameters to enable a visual comparison of diet A from study 1 ( - ) diet I from study $3(\cdot .-$. .) and diet K from study 4 (- . .). (a) Plasma phosphorus, (b) ionised calcium (iCa) and (c) parathyroid hormone (PTH). Diet A: $4.8 \mathrm{~g}$ total phosphorus $/ 4184 \mathrm{~kJ}$ (1000 kcal), $3.5 \mathrm{~g} \mathrm{P}$ from dihydrogen phosphate (SDHP)/4184 kJ (1000 kcal), calcium:phosphorus 0.56. Diet I: $3.86 \mathrm{~g}$ total phosphorus $/ 4184 \mathrm{~kJ}(1000 \mathrm{kcal}), 1.5 \mathrm{~g}$ phosphorus from SDHP/4184 kJ (1000 kcal), calcium:phosphorus 1.59. Diet K: $3.59 \mathrm{~g}$ total phosphorus $/ 4184 \mathrm{~kJ}(1000 \mathrm{kcal}), 1.5 \mathrm{~g}$ phosphorus from SDHP/4184 kJ (1000 kcal), calcium:phosphorus 0.93. Reference ranges are shown for each parameter.

compared with organic P sources (for 40-60\%), there are limited data clearly describing the influence of different dietary sources of $\mathrm{P}$ on the regulation of postprandial plasma $\mathrm{P}^{(3,8,47)}$. Finco et al. ${ }^{(8)}$ showed that cats fed a diet with $2 \cdot 1 \mathrm{~g}$ P from SDHP/4184 $\mathrm{kJ}(1000 \mathrm{kcal})$ showed an increase in plasma $\mathrm{P}$ concentration from just under $5 \mathrm{mg} / \mathrm{dl}(1.61 \mathrm{mmol} / \mathrm{l})$ to a concentration of $8 \mathrm{mg} / \mathrm{dl}(2.58 \mathrm{mmol} / \mathrm{l}) 2 \mathrm{~h}$ after feeding, which started to return towards baseline at $6 \mathrm{~h}$, compared with a $100 \%$ natural $\mathrm{P}$ source diet which did not change over $8 \mathrm{~h}$ post-meal.

Studies 3 and 4 suggest that diets formulated using SDHP $v$. STPP caused subtle differences in the postprandial plasma $\mathrm{P}$

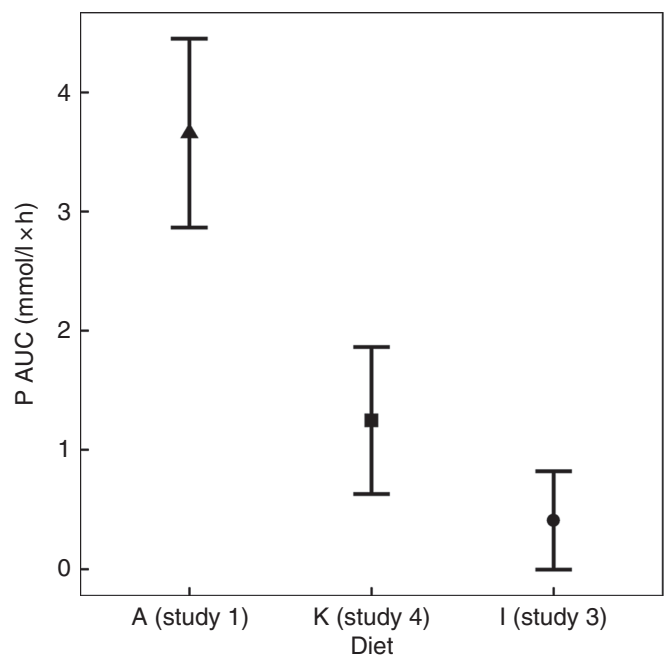

Fig. 10. AUC data to enable a visual comparison of $\operatorname{diet} A$ from study $1(\mathbf{A})$, diet I from study $3(0)$ and diet K from study $4(\square)$. Diet A: $4.8 \mathrm{~g}$ total phosphorus $/ 4184$ $\mathrm{kJ}$ (1000 kcal), $3.5 \mathrm{~g}$ phosphorus from sodium dihydrogen phosphate (SDHP)/ $4184 \mathrm{~kJ}(1000 \mathrm{kcal})$, calcium:phosphorus 0.56 . Diet l: $3.86 \mathrm{~g}$ total phosphorus/ $4184 \mathrm{~kJ}$ (1000 kcal), $1.5 \mathrm{~g}$ phosphorus from SDHP/4184 kJ (1000 kcal), calcium:phosphorus 1.59. Diet K: $3.59 \mathrm{~g}$ total phosphorus $/ 4184 \mathrm{~kJ}(1000 \mathrm{kcal})$, $1.5 \mathrm{~g}$ phosphorus from SDHP/4184 kJ (1000 kcal), calcium:phosphorus 0.93.

concentrations. This response is broadly in agreement with the findings reported by Karp et al. ${ }^{(25)}$, where ingestion of monophosphate and tripolyphosphate supplements by young healthy women resulted in similar changes in serum phosphate, but urinary $\mathrm{Ca}$ excretion reduced following the tripolyphosphate compared with the monophosphate supplement. This was suggested to be due to tripolyphosphate binding more Ca than the monophosphate supplement in the intestine ${ }^{(25)}$. The lack of difference between plasma kinetic profiles of mono- and tripolyphosphates may be due to monophosphates being the only form of $\mathrm{P}$ that can be absorbed by the intestine and cross into the bloodstream, according to data in serially killed rats ${ }^{(48)}$. The data shown here demonstrate that SDHP had a larger P AUC compared with that seen in STPP, suggesting that as a source of inorganic $\mathrm{P}$ it may have a greater impact on homoeostasis of plasma P than STPP in the cat. Whether this difference is biologically meaningful is unclear, but this finding warrants further investigation. In dogs, it has been shown that added inorganic $\mathrm{P}$ salts containing $\mathrm{Na}$ and $\mathrm{K}$ increase $2 \mathrm{~h}$ postprandial serum $\mathrm{P}$ and PTH above normal ranges ${ }^{(21)}$.

The bioavailability of dietary $\mathrm{P}$ is considered to be influenced not only by the P source and content of the diet but also by the $\mathrm{Ca}: \mathrm{P}$, with an increase in the Ca:P from around 1 to nearly 2 , reducing $\mathrm{P}$ digestibility and urinary $\mathrm{P}$ excretion ${ }^{(2,7,49-51)}$. To investigate the influence of $\mathrm{Ca}: \mathrm{P}$ on postprandial plasma $\mathrm{P}$ kinetics, a composite analysis of the present data was undertaken. This highlighted that diets $\mathrm{A}$ and $\mathrm{K}$ with a $\mathrm{Ca}: \mathrm{P}<1$ had a significantly higher plasma P AUC (Fig. 10) compared with those with a $\mathrm{Ca}: \mathrm{P}>1 \cdot 0$, with this response primarily resulting from a prolonged elevation in plasma P (Fig. 9). Interestingly, despite the remarkably similar total and added inorganic $\mathrm{P}$ levels between diets I and $\mathrm{K}$, plasma $\mathrm{P}$ returned to baseline within $3 \mathrm{~h}$ when cats were fed diet I with a Ca:P of 1.59 , adding further support that this response may have resulted from the 
difference in dietary $\mathrm{Ca}: \mathrm{P}$. The mechanism behind the reduction in bioavailability due to $\mathrm{Ca}: \mathrm{P}$ in diet stated in the literature is thought to be due to extra Ca being available to form insoluble, and hence non-bioavailable, $\mathrm{Ca}-\mathrm{Mg}$-phosphate complexes in the intestinal tract ${ }^{(50)}$. This insoluble complex formation is likely to be the reason that increasing $\mathrm{Mg}$ content of the diet has also been shown to decrease the percentage of $\mathrm{P}$ absorption and urinary $\mathrm{P}$ content ${ }^{(52)}$. The availability of $\mathrm{Ca}$ in the diet is also essential for its ability to form these insoluble complexes. Investigation into the differences between calcium chloride and calcium carbonate supplementation has found that calcium chloride had better capacity than calcium carbonate for reducing the presence of $\mathrm{P}$ in the urine, increasing retention ${ }^{(49)}$. It is important to note that it was not only the Ca:P that differed between diets I and $\mathrm{K}$; diet $\mathrm{K}$ also contained less crude fibre and had a negative food base excess (Table 2). Crude fibre increases faecal DM and bulk, which in turn can decrease apparent digestibility of $\mathrm{P}$ and other minerals ${ }^{(53)}$. The potential impact of the base excess of the diet has been studied in cats, where although there was no effect on plasma $\mathrm{P}$ concentrations or apparent digestibility of $\mathrm{P}$, the excretion of other minerals such as $\mathrm{Ca}$ are known to be affected by this and therefore could have contributed to some of the findings shown here ${ }^{(54,55)}$. Another factor that may alter the digestibility and absorption of $\mathrm{P}$ is the diet matrix. Diets based on wet chunk or dry kibble technologies (or format as stated in Table 1) may affect the availability of $\mathrm{P}$ through changes in transit times, most likely driven by differences in energy density between the matrices ${ }^{(56,57)}$. Visual assessment of data from diets $\mathrm{C}$ and $\mathrm{L}$, both wet chunk formats, compared with diet J, a dry kibble diet, suggests that plasma $\mathrm{P}$ starts to rise after $60 \mathrm{~min}$ in the dry format, while in wet plasma $\mathrm{P}$ was not significantly different to baseline at this time point. Aside from this difference, there appears to be little to distinguish between the diet formats in the small set of diets studied here. To draw further conclusions would require a comparison of more diets within the same study. One point to note is that diets for these studies were formulated using similar raw materials (within diet matrix) included at various ratios to alter the total $\mathrm{P}$ or $\mathrm{Ca}: \mathrm{P}$. As such, we have no information on whether other raw materials (such as alternative $\mathrm{Ca}$ or $\mathrm{P}$ sources) would have the same effect on plasma $\mathrm{P}$ concentrations.

Diets $\mathrm{A}$ and $\mathrm{K}$ have been shown to cause adverse effects on renal function in cats when fed over prolonged periods ${ }^{(20)}$. This observation is broadly in agreement with Dobenecker et al. ${ }^{(3,6)}$ who reported an increase in indicators of kidney dysfunction when a diet high in added inorganic $\mathrm{P}$ was fed and compared with a control diet low in both total and supplemented P. Diets A and K displayed a distinct kinetic profile compared with all the other diets tested, showing an extended elevation in plasma $\mathrm{P}$ across the $6 \mathrm{~h}$ postprandial period investigated. The differences in plasma $\mathrm{P}$ kinetic profiles, iCa and PTH concentrations observed between diets A and $\mathrm{K}$ suggest differences in the regulation of $\mathrm{P}$ homoeostasis. Differing levels of SDHP in the two diets apparently explain the higher peak in plasma $\mathrm{P}$ in cats fed diet $\mathrm{A}$, which also had a lower $\mathrm{Ca}: \mathrm{P}$ than diet K. These two factors may explain the marked increase in plasma $\mathrm{PTH}$, decrease in plasma iCa and increased urinary $\mathrm{P}$ excretion observed in cats fed diet A compared with diet K. Diet A also contained higher $\mathrm{Na}(3.33 \mathrm{~g} / 4184 \mathrm{~kJ}$ (1000 kcal) compared with $1.35 \mathrm{~g} / 4184 \mathrm{~kJ}(1000 \mathrm{kcal}))$ and less crude fibre $(5 \cdot 2 \mathrm{~g} / 4184 \mathrm{~kJ}$ (1000 kcal) compared with $7.49 \mathrm{~g} / 4184 \mathrm{~kJ}(1000 \mathrm{kcal}))$ than diet K. Increasing $\mathrm{Na}$ has been reported to increase $\mathrm{P}$ absorption of minerals through increasing $\mathrm{Na}^{+}$-dependent uptake of phosphate in rats ${ }^{(58)}$. In addition, diets with reduced crude fibre show increased absorption of minerals in humans ${ }^{(59)}$. With these differences in mind, it is surprising to note that when $5 \mathrm{~d}$ digestibility was measured by Alexander et $a l .{ }^{(20)}$ the mean apparent digestibility of $\mathrm{P}$ was $49.3 \%$ for diet $\mathrm{A}$ and $51.5 \%$ for diet $\mathrm{K}$ and may indicate that the dietary differences are not important for digestibility in this case. The subtle reduction in plasma $\mathrm{P}$ towards the end of this period in cats fed diet A indicates that P regulatory factors (e.g. PTH) are likely triggered and responding to the large $\mathrm{P}$ load in an attempt to return plasma $\mathrm{P}$ to normal. This somewhat mimics the response reported by Hazim et $a l .{ }^{(26)}$, when healthy men ingested a $500 \mathrm{mg}$ inorganic P supplement with a high fat meal. The sustained plasma P levels $6 \mathrm{~h}$ postprandially make it distinctly possible that plasma $\mathrm{P}$ may still be elevated at the time of the next feed, potentially exposing the kidneys and other tissues to incrementally higher $\mathrm{P}$ concentrations with each meal, which may explain the adverse effects that were observed when these diets were fed to cats over prolonged periods ${ }^{(20)}$. Consumption of a diet with a low Ca:P has been shown to cause nutritional hyperparathyroidism, resulting in enlargement of the parathyroid in cats, presumably as an attempt to maintain plasma Ca concentration ${ }^{(3)}$. The increase in PTH stimulates intestinal absorption of $\mathrm{Ca}$ and $\mathrm{P}$ and promotes urinary $\mathrm{P}$ excretion $^{(3)}$. A prolonged elevation in plasma $\mathrm{P}$ postprandially could also lead to increases in PTH production and thereby a reduction in plasma iCa. Increased FGF-23 production has been suggested to aid in maintaining $\mathrm{P}$ homoeostasis ${ }^{(1,21)}$. As suggested by data herein and Alexander et al. ${ }^{(20)}$, FGF-23 may respond to high elevated plasma P levels than PTH at a later stage. Studies have also shown that if FGF-23 levels remain high, there is a reduced PTH response to low plasma Ca concentrations, which could be a mechanism by which kidney damage may occur if highly available dietary $\mathrm{P}$ is consumed long-term ${ }^{(27)}$. Quantification of regulatory factors involved in $\mathrm{P}$ homoeostasis are therefore essential to understanding the potential impact of high levels of dietary $\mathrm{P}$.

In this study, inorganic $\mathrm{P}$ sources were found to dosedependently increase plasma $\mathrm{P}$ levels, similarly altering plasma $\mathrm{PTH}$; this response was apparently independent of total $\mathrm{P}$ and $\mathrm{Ca}: \mathrm{P}$. The exceptions to this peak PTH at 120 min were diet A, which was increased at $4 \mathrm{~h}$ post-meal as discussed above, and diet $\mathrm{K}$, which did not cause an increase in PTH during the trial day despite the sustained elevation of plasma P. In addition, in agreement with an earlier rat study, this increase in PTH occurred without a corresponding significant reduction in iCa between dietary manipulations, suggesting that plasma $\mathrm{P}$ may directly affect PTH production ${ }^{(60)}$. Although the plasma P data continued to show differences between diets in the studies following the proof of principle study, differences were not detected in either iCa or urinary excretion of $\mathrm{P}$. This may be due to the lower added inorganic $\mathrm{P}$ inclusion levels $(0-1.5 \mathrm{~g} / 4184 \mathrm{~kJ}$ (1000 kcal)) compared with diet A $(3.5 \mathrm{~g} / 4184 \mathrm{~kJ}(1000 \mathrm{kcal}))$. Consequently there may not have been sufficient stimulus, 
induced by added inorganic $\mathrm{P}$ to the diet, during the sampling day to induce a decrease in iCa and/or a significant increase in urinary $\mathrm{P}$ excretion. A lack of significant differences between diets for $24 \mathrm{~h}$ urinary $\mathrm{P}$ data may be due to two factors. First, no other diets studied in this programme provoked such a large increase in plasma P or PTH (nor a decrease in iCa) as diet A. This may indicate a threshold must be reached before any changes in urinary excretion of $\mathrm{P}$ are measurable. Second, the methodology used meant that there was no guarantee of an empty bladder before commencing the collection and no guarantee of the bladder being emptied before finishing the collection. As such it is possible that not all the excreted $\mathrm{P}$ was collected from all cats on the study day at every collection point. Interestingly, despite no measurable differences in $24 \mathrm{~h} \mathrm{P}$ excretion, diet $\mathrm{K}$ resulted in increased urinary excretion of $\mathrm{P}$ when fed over several weeks compared with the control group ${ }^{(20)}$. Thus, a single feed and a $24 \mathrm{~h}$ urine collection may not be enough to identify more subtle differences between diets. In summary, this also indicates that although this model is useful in showing differences between diets following the $6 \mathrm{~h}$ after a single feed, long-term feeding studies are required to determine the consequences of fluctuations in plasma P kinetics. From the data presented here, it is not possible to deduce whether similar kinetic profiles will be observed after a second meal or to extrapolate responses beyond the $6 \mathrm{~h}$ time frame. In addition, it should be recognised that firm conclusions around the mechanisms governing $\mathrm{P}$ homoeostasis may be limited due to the omission of markers of bone resorption and the methods employed during the $24 \mathrm{~h}$ urine collections.

In conclusion, the results of the present series of studies add to the growing literature, suggesting that inclusion of added inorganic $\mathrm{P}$ in diets results in a dose-dependent increase in plasma $\mathrm{P}$, with a concurrent elevation in $\mathrm{PTH}^{(26,29)}$. This relationship is far from straightforward, however, with a sustained postprandial elevation in plasma $\mathrm{P}$ apparent when diets with a $\mathrm{Ca}: \mathrm{P}<1.0$ were fed compared with that seen when the diet $\mathrm{Ca}: \mathrm{P}$ was $>1.5$. In addition, we present limited evidence that the source of added inorganic P can influence postprandial P kinetics, but further work is required to characterise the responses to other forms relevant to food manufacturing. In contrast, organic sources of $\mathrm{P}$ at the levels fed here do not appear to influence postprandial plasma $\mathrm{P}$ kinetics or $\mathrm{P}$ regulatory factors. Here, we present a model that can be applied to evaluate the short-term impact of a variety of dietary factors on short-term, postprandial regulation of plasma $\mathrm{P}$ homoeostasis. While some inferences to long-term feeding of the same diets have been made in the present manuscript, it is important to recognise that these data alone cannot infer the safety of a given diet composition.

\section{Acknowledgements}

The authors would like to acknowledge the skills and expertise of colleagues at WALTHAM in the care, training and sampling of the cats and for the analysis of samples collected throughout the studies. The authors recognise the contribution of Jonathan Elliott and Anna Riddle (The Royal Veterinary College, London) in the analysis of the PTH and FGF-23 samples.
This work was funded by Mars Petcare. The WALTHAM ${ }^{\oplus}$ Centre for Pet Nutrition is a fundamental research centre for Mars Petcare.

J. C. C., D. W. L., R. B. and P. W. conceived the project. J. C. C., G. T., M. G., D. W. L., R. B. and P. W. developed the overall research plan and had study oversight. J. C. C., G. T. and M. G. conducted the research and oversaw the analysis of the samples. R. S. and A. C. performed the statistical analysis. J. C. C. authored the paper with G. T., M. G., D. W. L., R. B. and P. W.

J. C. C., R. S., A. C., G. T., M. G., D. W. L., R. B. and P. W. were employees of Mars Petcare during the study.

\section{References}

1. Cooke A (2017) Dietary food-additive phosphate and human health outcomes. Compr Rev Food Sci Food Saf 16, 906-1021.

2. Kienzle E, Thielen C \& Pessinger C (1998) Investigations on phosphorus requirements of adult cats. $J$ Nutr 128, 2598s-2600s.

3. Pastoor FJ, Van't Klooster AT, Mathot JN, et al. (1995) Increasing phosphorus intake reduces urinary concentrations of magnesium and calcium in adult ovariectomized cats fed purified diets. J Nutr 125, 1334-1341.

4. Fettman MJ, Coble JM, Hamar DW, et al. (1992) Effect of dietary phosphoric acid supplementation on acid-base balance and mineral and bone metabolism in adult cats. Am J Vet Res 53, 2125-2135.

5. National Research Council (2006) National Research Council: Nutrient Requirements of Dogs and Cats. Washington, DC: National Academies Press.

6. Dobenecker B, Webel A, Reese S, et al. (2018) Effect of a high phosphorus diet on indicators of renal health in cats. J Feline Med Surg 20, 339-343.

7. Mack JK, Alexander LG, Morris PJ, et al. (2015) Demonstration of uniformity of calcium absorption in adult dogs and cats. J Anim Physiol Anim Nutr (Berl) 99, 801-809.

8. Finco DR, Barsanti JA \& Brown SA (1989) Influence of dietary source of phosphorus on fecal and urinary excretion of phosphorus and other minerals by male cats. AmJ Vet Res $\mathbf{5 0}$, 263-266.

9. Noori N, Sims J, Kopple J, et al. (2010) Organic and inorganic dietary phosphorus and its management in chronic kidney disease. Iran J Kidney Dis 4, 89-100.

10. Dobenecker B, Schaschl C, Webel A, et al. (2018) A comparison of the faecal and renal phosphorus excretion after excessive intake of monophosphate from calcium and potassium monophosphate in adult cats. In Proceedings of the Society of Nutrition Physiology, vol. 27, p. 95. Frankfurt am Main: DLG-Verlag.

11. Isakova T, Gutierrez O, Shah A, et al. (2008) Postprandial mineral metabolism and secondary hyperparathyroidism in early CKD. J Am Soc Nephrol 19, 615-623.

12. Silver J, Rodriguez M \& Slatopolsky E (2012) FGF23 and PTHdouble agents at the heart of CKD. Nephrol Dial Transplant 27, 1715-1720.

13. Nagode LA, Chew DJ \& Podell M (1996) Benefits of calcitriol therapy and serum phosphorus control in dogs and cats with chronic renal failure. Both are essential to prevent of suppress toxic hyperparathyroidism. Vet Clin North Am Small Anim Pract 26, 1293-1330.

14. Tani Y, Sato T, Yamanaka-Okumura H, et al. (2007) Effects of prolonged high phosphorus diet on phosphorus and calcium balance in rats. J Clin Biochem Nutr 40, 221-228.

15. Uribarri J (2007) Phosphorus metabolism and management in chronic kidney disease: phosphorus homeostasis in normal 
health and in chronic kidney disease patients with special emphasis on dietary phosphorus intake. Semin Dial 20, 295-301.

16. Kestenbaum B, Sampson JN, Rudser KD, et al. (2005) Serum phosphate levels and mortality risk among people with chronic kidney disease. J Am Soc Nephrol 16, 520-528.

17. Hitchman AJ, Hasany SA, Hitchman A, et al. (1979) Phosphate-induced renal calcification in the rat. Can J Physiol Pharmacol 57, 92-97.

18. Ritskes-Hoitinga J, Lemmens AG \& Beynen AC (1989) Nutrition and kidney calcification in rats. Lab Anim 23, 313-318.

19. Gonzalez-Parra E, Gracia-Iguacel C, Egido J, et al. (2012) Phosphorus and nutrition in chronic kidney disease. Int $J$ Nephrol 2012, 597605.

20. Alexander J, Stockman J, Atwal J, et al. (2018) Effects of the long term feeding of diets enriched with inorganic phosphorus on the adult feline kidney and phosphorus metabolism. BrJ Nutr (In the Press).

21. Dobenecker B \& Siedler S (2017) Knowing the total amount of phosphorus in a diet is not enough - different sources have different effects. In Congress of the European College of Veterinary Internal Medicine - Companion Animals. Malta: European Society of Veterinary Internal Medicine.

22. Berndt T \& Kumar R (2009) Novel mechanisms in the regulation of phosphorus homeostasis. Physiology 24, 17-25.

23. Moe SM, Zidehsarai MP, Chambers MA, et al. (2011) Vegetarian compared with meat dietary protein source and phosphorus homeostasis in chronic kidney disease. Clin J Am Soc Nephrol 6, 257-264.

24. Moore LW, Nolte JV, Gaber AO, et al. (2015) Association of dietary phosphate and serum phosphorus concentration by levels of kidney function. Am J Clin Nutr 102, 444-453.

25. Karp HJ, Vaihia KP, Kärkkäinen MUM, et al. (2007) Acute effects of different phosphorus sources on calcium and bone metabolism in young women: a whole-foods approach. Calcif Tissue Int 80, 251-258.

26. Hazim J, Hlais S, Ghattas H, et al. (2014) Phosphorus supplement alters postprandial lipemia of healthy male subjects: a pilot cross-over trial. Lipids Health Dis 13, 109.

27. Wetmore JB, Santos PW, Mahnken JD, et al. (2011) Elevated FGF23 levels are associated with impaired calcium-mediated suppression of PTH in ESRD. J Clin Endocrinol Metab 96, E57-E64.

28. Canalejo R, Canalejo A, Martinez-Moreno JM, et al. (2010) FGF23 fails to inhibit uremic parathyroid glands. J Am Soc Nephrol 21, 1125-1135.

29. Siedler S \& Dobenecker B (2015) Effect of different P sources in high phosphorus diets with balanced $\mathrm{Ca} / \mathrm{P}$ ratio on serum PTH, P and calcium levels as well as apparent digestibility of these minerals in dogs. Conference of the European Society of Veterinary Internal Medicine, Toulouse.

30. Bjornvad CR, Nielsen DH, Armstrong PJ, et al. (2011) Evaluation of a nine-point body condition scoring system in physically inactive pet cats. Am J Vet Res 72, 433-437.

31. Geddes R, Finch N, Elliott J, et al. (2013) Fibroblast growth factor 23 in feline chronic kidney disease.J Vet Intern Med 27, 234-241.

32. Williams T, Elliott J \& Syme H (2012) Calcium and phosphate homeostasis in hyperthyroid cats-associations with development of azotaemia and survival time. J Small Anim Pract 53, 561-571.

33. $\mathrm{R}$ Development Core Team (2017) $R$ : A Language and Environment for Statistical Computing. Vienna: R Foundation for Statistical Computing.

34. Bates D, Mächler M, Bolker B et al. (2015) Fitting linear mixed-effects models using lme4. 67, 1-48.

35. Hothorn T, Bretz F \& Westfall P (2008) Simultaneous inference in general parametric models. Biom J 50, 346-363.
36. Wickham H (2009) Ggplot2: Elegant Graphics for Data Analysis, vol. 16. New York: Springer Verlag.

37. Rosol TJ \& Capen CC (1996) Pathophysiology of calcium, phosphorus, and magnesium metabolism in animals. Vet Clin North Am Small Anim Pract 26, 1155-1184.

38. Kemi VE, Karkkainen MU \& Lamberg-Allardt CJ (2006) High phosphorus intakes acutely and negatively affect $\mathrm{Ca}$ and bone metabolism in a dose-dependent manner in healthy young females. Br J Nutr 96, 545-552.

39. Kärkkäinen M \& Lamberg-Allardt C (1996) An acute intake of phosphate increases parathyroid hormone secretion and inhibits bone formation in young women. J Bone Miner Res 11, 1905-1912.

40. McDonald JL Jr, Stookey GK \& Muhler JC (1970) Radiophosphorus metabolism of different phosphate compounds in experimental animals. J Dent Res 49, 1042-1048.

41. Geddes RF, Biourge V, Chang Y, et al. (2016) The effect of moderate dietary protein and phosphate restriction on calcium-phosphate homeostasis in healthy older cats. $J$ Vet Intern Med 30, 1690-1702.

42. Finch NC, Geddes RF, Syme HM, et al. (2013) Fibroblast growth factor 23 (FGF-23) concentrations in cats with early nonazotemic chronic kidney disease (CKD) and in healthy geriatric cats. J Vet Intern Med 27, 227-233.

43. Geddes RF, Elliott J \& Syme HM (2013) The effect of feeding a renal diet on plasma fibroblast growth factor 23 concentrations in cats with stable azotemic chronic kidney disease. $J$ Vet Intern Med 27, 1354-1361.

44. Geddes RF, Elliott J \& Syme HM (2015) Relationship between plasma fibroblast growth factor-23 concentration and survival time in cats with chronic kidney disease. J Vet Intern Med 29, 1494-1501.

45. Nishida Y, Taketani Y, Yamanaka-Okumura H, et al. (2006) Acute effect of oral phosphate loading on serum fibroblast growth factor 23 levels in healthy men. Kidney Int $\mathbf{7 0}$, 2141-2147.

46. Takasugi S, Akutsu M \& Nagata M (2014) Oral phosphorus supplementation secondarily increases circulating fibroblast growth factor 23 levels at least partially via stimulation of parathyroid hormone secretion. J Nutr Sci Vitaminol 60, 140-144.

47. Calvo MS, Moshfegh AJ \& Tucker KL (2014) Assessing the health impact of phosphorus in the food supply: issues and considerations. Adv Nutr 5, 104-113.

48. Fingerhut M, Ruf F \& Lang K (1966) Zur Frage der Resorption von Polyphosphaten (On the question of the absorption of polyphosphates). Zeitschrift für Ernährungswissenschaft $\mathbf{6}$, 228-241.

49. Pastoor FJ, Opitz R, Van't Klooster AT, et al. (1994) Dietary calcium chloride vs. calcium carbonate reduces urinary $\mathrm{pH}$ and phosphorus concentration, improves bone mineralization and depresses kidney calcium level in cats. J Nutr $\mathbf{1 2 4}$, 2212-2222

50. Pastoor FJ, Van't Klooster AT, Mathot JN, et al. (1994) Increasing calcium intakes lower urinary concentrations of phosphorus and magnesium in adult ovariectomized cats. J Nutr 124, 299-304.

51. Kienzle E, Pessinger C \& Thielen C (1998) Phosphorus requirements of adult cats. J Anim Physiol Anim Nutr 80, 90-100.

52. Pastoor FJ, Van't Klooster AT, Opitz R, et al. (1995) Effect of dietary magnesium level on urinary and faecal excretion of calcium, magnesium and phosphorus in adult, ovariectomized cats. Br J Nutr 74, 77-84.

53. Prola L, Dobenecker B, Mussa PP, et al. (2010) Influence of cellulose fibre length on faecal quality, mineral excretion and 
nutrient digestibility in cat. J Anim Physiol Anim Nutr 94 362-367.

54. Ching SV, Fettman MJ, Hamar DW, et al. (1989) The effect of chronic dietary acidification using ammonium chloride on acid-base and mineral metabolism in the adult cat. J Nutr 119, 902-915.

55. Kienzle E, Schuknecht A \& Meyer H (1991) Influence of food composition on the urine $\mathrm{pH}$ in cats. J Nutr 121, S87-S88.

56. Coradini M, Rand JS, Filippich LJ, et al. (2015) Associations between meal size, gastric emptying and post-prandial plasma glucose, insulin and lactate concentrations in meal-fed cats. J Anim Physiol Anim Nutr 99, 757-766.

57. Vist GE \& Maughan RJ (1995) The effect of osmolality and carbohydrate content on the rate of gastric emptying of liquids in man. J Physiol 486, 523-531.
58. Marks J, Lee GJ, Nadaraja SP, et al. (2015) Experimental and regional variations in $\mathrm{Na}^{+}$-dependent and $\mathrm{Na}^{+}$-independent phosphate transport along the rat small intestine and colon. Physiol Rep 3, e12281.

59. Reinhold JG, Faradji B, Abadi P, et al. (1976) Decreased absorption of calcium, magnesium, zinc and phosphorus by humans due to increased fiber and phosphorus consumption as wheat bread. J Nutr 106, 493-503.

60. Hernández A, Concepción MT, Rodríguez M, et al. (1996) High phosphorus diet increases preproPTH mRNA independent of calcium and calcitriol in normal rats. Kidney Int $\mathbf{5 0}$, 1872-1878.

61. Jeremias JT, Nogueira SP, Brunetto MA, et al. (2013) Predictive formulas for food base excess and urine $\mathrm{pH}$ estimations of cats. Anim Feed Sci Technol 182, 82-92. 